\title{
Conjugacy in Garside groups II: structure of the ultra summit set
}

\author{
Joan S. Birman*, Volker Gebhardt and Juan González-Meneses**
}

\begin{abstract}
This paper is the second in a series in which the authors study the conjugacy decision problem (CDP) and the conjugacy search problem (CSP) in Garside groups.

The ultra summit set $\operatorname{USS}(X)$ of an element $X$ in a Garside group $G$ is a finite set of elements in $G$, which is a complete invariant of the conjugacy class of $X$ in $G$. A fundamental question, if one wishes to find bounds on the size of $\operatorname{USS}(X)$, is to understand its structure. In this paper we introduce two new operations on elements $Y \in \mathrm{USS}(X)$, called 'partial cycling' and 'partial twisted decycling', and prove that if $Y, Z \in \operatorname{USS}(X)$, then $Y$ and $Z$ are related by sequences of partial cyclings and partial twisted decyclings. These operations are a concrete way to understand the minimal simple elements whose existence follows from the convexity property of ultra summit sets.

Using partial cycling and partial twisted decycling, we investigate the structure of a directed graph $\Gamma_{X}$ determined by USS $(X)$, and show that $\Gamma_{X}$ can be decomposed into 'black' and 'grey' subgraphs. There are applications relating to the authors' program for finding a polynomial solution to the CDP/CSP in the case of braids, which is outlined in the first paper of this series. A different application is to give a new algorithm for solving the CDP/CSP in Garside groups which is faster than all other known algorithms, even though its theoretical complexity is the same as that of the established algorithm using ultra summit sets. There are also applications to the theory of reductive groups.
\end{abstract}

Mathematics Subject Classification (2000). 20F36, 20F10, $20 \mathrm{E} 45$.

Keywords. Garside groups, conjugacy problem, ultra summit set, partial cycling.

\section{Contents}

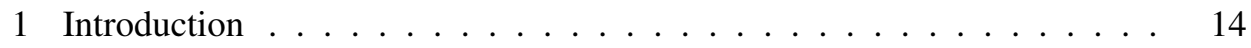

1.1 Definitions and basic facts . . . . . . . . . . . 15

2 The structure of the ultra summit set . . . . . . . . . . . 20

\footnotetext{
${ }^{*}$ Corresponding author. Partially supported by the U.S.National Science Foundation, under Grant DMS-0405586.

** Partially supported by MTM2004-07203-C02-01 and FEDER.
} 
2.1 Description of the minimal simple elements . . . . . . . . . 20

2.2 Black and grey components of $\operatorname{USS}(X) \ldots \ldots \ldots$

2.3 Intersection of black and grey components . . . . . . . . . 30

3 Rigid elements . . . . . . . . . . . . . . . . . . . 33

3.1 Vertices and arrows in the USS graph of a rigid element . . . . . 33

3.2 Paths in the USS graph of a rigid element . . . . . . . . . . . 37

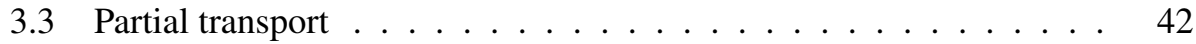

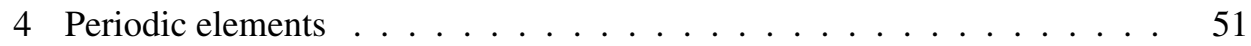

5 Applications .......................... 53

5.1 Complexity of the CDP/CSP for pseudo-Anosov and periodic braids 53

5.2 A new solution to the CDP/CSP in Garside groups . . . . . . . . 57

5.3 Partial cycling subsumes decycling . . . . . . . . 58

5.4 An application to the theory of reductive groups $\ldots \ldots \ldots$

\section{Introduction}

Garside groups were introduced by P. Dehornoy and L. Paris in [11]. Roughly speaking, they are groups that satisfy the same kind of properties that were shown to hold in Artin's braid groups $\left\{B_{n}, n=1,2, \ldots\right\}$ in the foundational paper of F. Garside [17], and soon afterwards in spherical Artin-Tits groups by Brieskorn and Saito [7]. More precisely, they are groups admitting a lattice structure, invariant under left multiplication, and satisfying some additional conditions. The focus of this paper and of its companions [3] and [4] is on gaining an improved understanding of the known algorithmic solutions in Garside groups of the conjugacy decision problem (CDP), that is, to decide, for arbitrary $X, Y \in G$ whether there exists $Z$ such that $Y=Z^{-1} X Z$; and also the conjugacy search problem (CSP), that is, to find $Z$ as above when $X$ and $Y$ are known to be conjugate.

In the manuscript [18] the second author of this paper introduced a complete invariant of the conjugacy class of an arbitrary element $X$ in an arbitrary Garside group $G$, which is a certain finite set of elements, called the 'ultra summit set' $\operatorname{USS}(X)$ (Definition 1.9.) For arbitrary $X, Y \in G$ either $\operatorname{USS}(X)=\operatorname{USS}(Y)$ (in which case $X$ is conjugate to $Y$ ) or $\operatorname{USS}(X) \cap \operatorname{USS}(Y)=\emptyset$. From this it follows that in order to determine whether $X$ and $Y$ are conjugate, one must compute the entire set $\operatorname{USS}(X)$ and a single element in USS $(Y)$. That leads one immediately to ask about bounds on the number of elements in $\operatorname{USS}(X)$. This paper is a first step toward answering that question. The focus of this paper is on the structure of $\operatorname{USS}(X)$, a matter which we isolated as being a key issue as we worked toward a polynomial algorithm for the $\mathrm{CDP} / \mathrm{CSP}$ in $B_{n}$. See $\S 1.4$ of [3] for an outline of our approach to that problem, and a discussion of how the work in this paper fits into that program and the open problems that remain. 
Here is a brief overview of the contents of this paper. In $\S 1.1$ we give a quick review of the necessary background, with all essential definitions. In $\$ 2$ we will describe new properties of the structure of ultra summit sets, showing that in order to compute them one only needs to apply two special kinds of conjugation, which we call 'partial cycling' and 'partial twisted decycling' (Definition 2.9). Using it, we discover new aspects of the combinatorics that are both closely related to things we already understand well, but at the same time present new challenges. We will study the graph $\Gamma_{X}$ which describes $\operatorname{USS}(X)$ of an element $X \in G$, a Garside group, and prove that $\Gamma_{X}$ splits into two subgraphs, which we call 'black' and 'grey'. The similarities and differences between the graphs $\Gamma_{X}$ and $\Gamma_{X^{-1}}$ are particularly interesting. In $\S 2$ we also give several key examples which illustrate our new discoveries.

In $\S 3$ and $\S 4$ we examine the effects of partial cycling on two special kinds of elements in a Garside group $G$ : rigid elements and periodic elements. In this regard we note that, while the work in this paper is applicable to all Garside groups and no assumptions are made that restrict attention to the braid groups $B_{n}$, the reader who is familiar with the Thurston-Nielsen trichotomy (see $\$ 1.3$ of [3]) in braid groups and with the main result in [3] will recognize that rigid elements and periodic elements have particular importance in the case of braids. This is relevant for the program that we outlined in $\$ 1.4$ of [3] for studying the CDP/CSP in the braid groups.

The final section, $\S 5$ is devoted to applications. The first application, in $\S 5.1$, is to show how our new knowledge of the structure of $\operatorname{USS}(X)$ in $\S 3$ and $\S 4$ advances our efforts to find a polynomial solution to the CDP/CSP in the special case of the braid groups. Unfortunately, we were unable to find a bound for the number of elements in $\operatorname{USS}(X)$ in this paper, even when we restrict to rigid elements. Finding such a bound is open question 2 of $\$ 1.4$ of [3], and remains for future work. Examples are given in $\$ 5.1$ that illustrate the remaining difficulties. Such examples would have been impossible to understand without the work in this paper. We then discuss the periodic case, briefly. A polynomial solution to the CDP/CSP for periodic braids will be given in the next paper in this series, [4].

The second application, in $\S 5.2$ is to develop a new solution to the CDP/CSP in arbitrary Garside groups. This new algorithm is faster than the one in [18], although its theoretical complexity is essentially the same. The third application, in $\$ 5.3$ is to show that, in a sense, partial cycling subsumes cycling. This is probably more of theoretical interest than of interest for complexity. The final application, in $\S 5.4$, is a brief note to mention that, as was communicated to us by Jean Michel, our work on the structure of the USS $(X)$ of a periodic element has implications for the theory of reductive groups. We refer to [12] for details.

1.1. Definitions and basic facts. Our review of what we need from the literature will be brief. We strongly recommend that non-experts consult $\$ 1$ of [3] for a more leisurely introduction to Garside groups and a review of what is known about the CDP/CSP with lots of examples. 
Definition 1.1. A group $G$ is said to be a Garside group (of finite type) if it satisfies the following three properties:

(A) $G$ admits a lattice order $(G, \preccurlyeq, \vee, \wedge)$, invariant under left-multiplication.

This means in particular that, with respect to the order $\preccurlyeq$, every pair of elements $s, t \in G$ admits a unique $\operatorname{lcm} s \vee t$ and a unique gcd $s \wedge t$. Let $P=\{p \in$ $G ; 1 \preccurlyeq p$. Then $P \subset G$ is a monoid. We call the elements of $P$ the positive elements of $G$.

(B) There exists an element $\Delta \in P$, called the Garside element, satisfying two properties: (a) The interval $[1, \Delta]=\{s \in G ; 1 \preccurlyeq s \preccurlyeq \Delta\}$ is finite and generates $G$; (b) $\Delta^{-1} P \Delta=P$.

(C) The monoid $P$ is atomic, that is, for all $X \in P$ there exists an upper bound on the length of a chain $1 \prec X_{1} \prec \cdots \prec X_{n}=X$. The atoms are those elements $a \in P$ for which $u \in P, \quad 1 \neq u \preccurlyeq a \Longrightarrow u=a$. The atoms are in $[1, \Delta] \subset P$ and they generate $G$.

Many examples of Garside groups can be found in [3].

Definition 1.2. The elements in $[1, \Delta]$ are called the simple elements of $G$. A prefix of $x \in G$ is an element $q \in G$ such that $q^{-1} x \in P$. A simple prefix $q$ of $x$ is a prefix of $x$ which is simple.

Definition 1.3. Given $X \in G$, we say that a decomposition of $X$ is in left normal form:

$$
X=\Delta^{p} x_{1} \ldots x_{r} \quad(r \geq 0)
$$

if $p \in \mathbb{Z}$ is the maximal power such that $\Delta^{p} \preccurlyeq X$, and $x_{i} \neq 1$ is the maximal simple prefix of $x_{i} x_{i+1} \ldots x_{r}$ for every $i=1, \ldots, r$. A pair of simple elements $a, b \in[1, \Delta]$ is left weighted if the product $a b$ is in left normal form as written. If $X=\Delta^{p} x_{1} \ldots x_{r}$ is in left normal form, the infimum, supremum and canonical length of $X$, are defined by $\inf (X)=p, \sup (X)=p+r$ and $\ell(X)=r$, respectively.

Definition 1.4. The symbol $\tau$ denotes the inner automorphism of $G$ that is defined by $\tau(x)=\Delta^{-1} x \Delta$. It is called the twisting automorphism.

Definition 1.5. If $u, v \in G$ and $u v=\Delta$, then $v=\partial(u)$. It is a basic property of the lattice structure on $G$ that the map $u \rightarrow \partial(u)$ is a permutation of the finite set of simple elements. Therefore if $u$ is simple and $v=\partial(u)$, it makes sense to say that $u=\partial^{-1}(v)$.

There are two special types of conjugations that have been recognized, ever since the work of Elrifai and Morton in [14], to be of fundamental importance in the combinatorics that underlie all of our work: 
Definition 1.6. If $\ell(X)>0$ and has left normal form as in (1), then cycling $\mathbf{c}(X)$ and decycling $\mathbf{d}(X)$ are the special conjugates of $X$ that are defined by:

$$
\mathbf{c}(X)=\Delta^{p} x_{2} \ldots x_{r} \tau^{-p}\left(x_{1}\right) \quad \text { and } \quad \mathbf{d}(X)=x_{r} \Delta^{p} x_{1} \ldots x_{r-1}
$$

We now define twisted decycling of $X$ to mean $\tau(\mathbf{d}(X))$.

Twisted decycling will be important later. To see how cycling and decycling enter into the picture, recall the following definition [14]:

Definition 1.7. The super summit set $\operatorname{SSS}(X)$ of an element $X \in G$ is the set of all elements $Y$ in the conjugacy class of $X$ with the property that if $Y=\Delta^{q} y_{1} \ldots y_{s}$ in left normal form, then $q=\inf (Y)$ is maximal and $q+s=\sup (Y)$ is minimal.

Remark 1.8. It is known that $\operatorname{SSS}(X)$ is always non-empty and finite and that $\operatorname{SSS}(X)$ consists of the conjugates of $X$ having minimal canonical length [14, Corollary 4.4].

Let us also recall (e.g. [14, proof of Proposition 4.5]) that if $X$ has left normal form as in (1), then the left normal form of $X^{-1}$ is determined by that of $X$ :

$$
X^{-1}=\Delta^{-p-r} x_{r}^{\prime} \ldots x_{1}^{\prime}, \text { where } x_{i}^{\prime}=\tau^{-p-i}\left(\partial\left(x_{i}\right)\right) .
$$

In particular, $\ell\left(Y^{-1}\right)=\ell(Y)$ for arbitrary $Y$, which implies that $\operatorname{SSS}\left(X^{-1}\right)=$ $\left\{y^{-1} \mid y \in \operatorname{SSS}(X)\right\}$.

This interplay between $X$ and $X^{-1}$ will play an important role in our work.

See Theorem 1.10 of [3] and the discussion that precedes it for a description of how cycling and decycling enable one, for any $X \in G$, to compute at least one element $\tilde{X} \in \operatorname{SSS}(X)$. However, we will not be working with $\operatorname{SSS}(X)$ in this paper. Instead, we consider a subset of $\operatorname{SSS}(X)$ which was introduced by the second author of this paper in [18].

Definition 1.9. The ultra summit set $\operatorname{USS}(X)$ of an element $X \in G$ is the subset of elements $Y \in \operatorname{SSS}(X)$ such that $\mathbf{c}^{m}(Y)=Y$, for some $m>0$.

Note that $\operatorname{USS}(X)$ is always non-empty, because if $Y \in \operatorname{SSS}(X)$, then $\mathbf{c}(Y)$ gives us another element of $\operatorname{SSS}(X)$, and since $\operatorname{SSS}(X)$ is finite, it follows that after some number of iterations of cycling we will find integers $m_{1}, m_{2}$, with $m_{1}<m_{2}$, such that $\mathbf{c}^{m_{1}}(Y)=\mathbf{c}^{m_{2}}(Y)$. But then $\mathbf{c}^{m_{1}}(Y)$ is in $\operatorname{USS}(X)$.

Definition 1.10. Given $X \in G$ and $Y \in \operatorname{USS}(X)$, we say that a simple element $s \neq 1$ is a minimal simple element for $Y$ with respect to $\operatorname{USS}(X)$ if $Y^{s}=s^{-1} Y s \in \operatorname{USS}(X)$, and no proper prefix of $s$ satisfies this property. 
Remark 1.11. If $X \in G$ and $Y, Y^{u} \in \operatorname{USS}(X)$ for a positive element $u$ such that $s$ is a minimal simple element for $Y$ satisfying $s \preccurlyeq u$, it is clear by induction that $u$ admits a decomposition $u=s_{1} \ldots s_{m}$ as a product of minimal simple elements such that $s_{1}=s$.

In [18] it is shown how to compute the minimal simple elements corresponding to a given $Y \in \operatorname{USS}(X)$, and this allows one to compute the whole $\operatorname{USS}(X)$, starting with a single element $\widetilde{X} \in \operatorname{USS}(X)$. To state the theorem that we will use from [18], we need one more concept:

Definition 1.12. Given $X \in G$, there is a directed graph, $\Gamma_{X}$, which describes the entire set $\operatorname{USS}(X)$, and is defined by the following data:

(1) Each vertex represents an element $Y \in \operatorname{USS}(X)$.

(2) For every $Y \in \operatorname{USS}(X)$ and every minimal simple element $s$ for $Y$ with respect to $\operatorname{USS}(X)$, there is an arrow labeled by $s$ going from $Y$ to $Y^{s}$.

The algorithm in [18] computes the graph $\Gamma_{X}$ using the following 'convexity theorem', which is analogous to a related convexity theorem proved for super summit sets in [16]. It will be seen to be of fundamental importance in our work, and will be given concrete meaning in many examples.

Theorem 1.13 ([18, Theorem 1.18 and Corollary 1.19]). Let $X \in G$ and $Y \in$ $\operatorname{USS}(X)$.

(i) If $s, t \in G$ are such that $Y^{s} \in \operatorname{USS}(X)$ and $Y^{t} \in \operatorname{USS}(X)$, then $Y^{s \wedge t} \in$ $\operatorname{USS}(X)$.

(ii) For every $u \in P$ there is a unique element $c_{Y}(u)$ which is minimal with respect to $\preccurlyeq$ among the elements satisfying $u \preccurlyeq c_{Y}(u)$ and $Y^{c_{Y}(u)} \in \operatorname{USS}(X)$.

(iii) The graph $\Gamma_{X}$ that is described in Definition 1.12 is connected.

Notice that the set of minimal simple elements for $Y$ is contained in $c_{Y}(A)=$ $\left\{c_{Y}(a) \mid a\right.$ is an atom $\}$, hence the number of minimal simple elements for $Y$ is bounded by the number of atoms. In the case of $B_{n}$, with its classical Garside structure, there are $n-1$ atoms, so in $\Gamma_{X}$ there are at most $n-1$ arrows starting at a given vertex.

We remark that one obtains an element in $\operatorname{USS}(X)$ by iterated application of cycling to an element in $\operatorname{SSS}(X)$. The number of times one needs to apply cycling, in order to go from an element in $\operatorname{SSS}(X)$ to an element in $\operatorname{USS}(X)$ is not known in general. That is open question 3 in $\$ 1.4$ of [3]. Nevertheless, the theoretical complexity of the algorithm in [18] is not worse than the theoretical complexity of the algorithm in [16], which is based upon $\operatorname{SSS}(X)$, and the performance of the algorithm is substantially better in practice. Later in this paper, in $\S 5.2$, we will obtain yet another 
algorithm which is faster than the one in [18], although its theoretical complexity is unchanged.

The conjugating elements involved in cycling or decycling will play a crucial role in our work (as they did in [3]), so to avoid repeated references to the automorphism $\tau$ we introduce convenient shorthand:

Definition 1.14. Given $X \in G$ whose left normal form is $X=\Delta^{p} x_{1} \ldots x_{r}(r>0)$, we define the initial factor of $X$ as $\iota(X)=\tau^{-p}\left(x_{1}\right)$, and the final factor of $X$ as $\varphi(X)=x_{r}$. If $r=0$ we define $\iota\left(\Delta^{p}\right)=1$ and $\varphi\left(\Delta^{p}\right)=\Delta$. Equivalently $\iota(X)=X \Delta^{-p} \wedge \Delta$ and $\varphi(X)=\left(\Delta^{p+r-1} \wedge X\right)^{-1} X$.

Rigid elements in a Garside group were introduced in [3]:

Definition 1.15. Let $X=\Delta^{p} x_{1} \ldots x_{r}$ be in left normal form, with $r=\ell(X)>0$. Then $X$ is rigid if the element $\Delta^{p} x_{1} \ldots x_{r} \tau^{-p}\left(x_{1}\right)$ is in left normal form as written.

Remark 1.16. Notice that if $X$ is rigid, then the cycling of $X$, that is, $\mathbf{c}(X)=$ $\Delta^{p} x_{2} \ldots x_{r} \tau^{-p}\left(x_{1}\right)$ is in left normal form as written. Actually, this latter property is equivalent to $X$ being rigid if $r>1$. But we prefer the definition above, otherwise every element of canonical length 1 would be rigid.

Proposition 1.17 ([3, Proposition 3.2]). The following are equivalent characterizations of rigid elements. (i) $X$ is rigid. (ii) $\ell(X)>0$ and $\varphi(X) \iota(X)$ is left weighted as written. In particular normal forms are preserved under cycling. (iii) $\ell(X)>0$ and $\iota(X) \wedge \iota\left(X^{-1}\right)=1$.

Remark 1.18. The third condition implies that $X$ is rigid if and only if $X^{-1}$ is rigid. It is important to notice that the assumption $\ell(X)>0$ implies that $\Delta$ and its powers are not rigid.

Remark 1.19. We need one more set of ideas from the existing literature before we can begin our new work. In $\S 5.1$ we will specialize our work about Garside groups to the braid groups $B_{n}$. In addition, all of our examples will be taken from braid groups. It therefore is important to keep in mind that the braid group $B_{n}$ acts on the $n$-times punctured disc $D_{n}$, and that this action determines a faithful representation of $B_{n}$ as the mapping class group of $D_{n}$, that is, the group $\pi_{0}\left(\operatorname{Diff}^{+}\left(D_{n}\right)\right)$. The reason this is important is that, as a consequence, the well-known Thurston-Nielsen Trichotomy, introduced by Thurston in [22] applies: braids come in three flavors: pseudo-Anosov (or PA), periodic and reducible. We refer the reader to [22] for the foundational paper in this area and to [3] for a very brief description of the trichotomy. We will not discuss it here because we use it in a very peripheral way: to identify our examples as being PA or periodic. We note, however, that in [3] the authors of this paper proved 
the important fact that every PA braid in its USS has a 'small' rigid power, so that all results about rigid elements in Garside groups apply ipso-facto to powers of PA braids. A major part of the work in this paper, notably the work in $\S 3$ concerns rigid elements in Garside groups. As a result, much of the work in this paper has special relevance in the key case of PA braids.

Acknowledgements. The authors thank Jean Michel, who showed us the importance of partial cyclings in the study of reductive groups, and encouraged us to show the results in $\S 4$.

J. Birman thanks the Project MTM2004-07203-C02-01 of the Spanish Ministerio de Ciencia y Tecnología for hosting her visit to Seville in November 2004, so that she and J. Gonzalez-Meneses could work together on this project.

J. González-Meneses thanks the Project MTM2004-07203-C02-01 of the Spanish Ministerio de Ciencia y Tecnología and the Columbia University Department of Mathematics for hosting his two visits to New York, in July 2004 and March-April 2006.

V. Gebhardt thanks the Departamento de Álgebra de la Universidad de Sevilla and the Junta de Andalucía, for funding his visit to Seville in January 2006.

\section{The structure of the ultra summit set}

In this section we begin the new work in this paper. In $\$ 2.1$ we begin to investigate the minimal simple elements whose existence was established in statement (ii) of Theorem 1.13. In particular, in Theorem 2.5 we give new meaning to the somewhat elusive element $c_{Y}(u)$. Using our description, we introduce partial cycling and partial twisted decycling in Definition 2.9. The main result in $\$ 2.1$ is Corollary 2.10. As a result of this corollary, we will prove that $\Gamma_{X}, X \in G$ splits into two (in general not connected) subgraphs which we call 'grey' and 'black'. The properties of the grey and black subgraphs are studied in $\$ 2.2$ and $\$ 2.3$.

2.1. Description of the minimal simple elements. As was proved in [3], the initial and final factors $\iota(X), \varphi(X)$ and $\iota\left(X^{-1}\right), \varphi\left(X^{-1}\right)$ are closely related:

Lemma 2.1 ([3, Lemma 1.8]). For $X \in G$ one has $\iota\left(X^{-1}\right)=\partial(\varphi(X))$ and $\varphi\left(X^{-1}\right)=$ $\partial^{-1}(\iota(X))$.

We saw in Theorem 1.13 that, given one element $X \in \operatorname{USS}(X)$, one can obtain any other element in USS $(X)$ just through conjugations by minimal simple elements. In this section we will describe the minimal simple elements for $X \in \operatorname{USS}(X)$, and show how they determine the graph $\Gamma_{X}$. See Corollary 2.7 and the examples which follow it. These examples will be used throughout the paper. 
Lemma 2.2. Given $X \in G$ with $\ell(X)>0$, one has:

$$
X^{\iota(X)}=\mathbf{c}(X), \quad X^{\varphi(X)^{-1}}=\mathbf{d}(X), \quad X^{\iota\left(X^{-1}\right)}=X^{\partial(\varphi(X))}=\tau(\mathbf{d}(X)) .
$$

Proof. The first two claims follow from the definitions. The third one is shown as follows: $X^{\partial(\varphi(X))}=X^{\varphi(X)^{-1} \Delta}=(\mathbf{d}(X))^{\Delta}=\tau(\mathbf{d}(X))$.

Remark 2.3. By the above result, the cycling and the twisted decycling of $X$ are conjugates of $X$ by simple elements. Moreover, the twisted decycling of $X$ corresponds to a cycling of $X^{-1}$, since it is a conjugation by $\iota\left(X^{-1}\right)$. In the same way, the cycling of $X$ corresponds to a twisted decycling of $X^{-1}$.

Lemma 2.4. The twisting automorphism $\tau$ commutes with both cycling and decycling. Moreover, the USS of an element is closed under twisting, cycling and decycling.

Proof. It is clear from the definitions that $\iota(\tau(X))=\tau(\iota(X))$ and $\varphi(\tau(X))=$ $\tau(\varphi(X))$. In particular, $\mathbf{c}(\tau(X))=\tau(X)^{\tau(\iota(X))}=\tau\left(X^{\iota(X)}\right)=\tau(\mathbf{c}(X))$, and $\mathbf{d}(\tau(X))=\tau(X)^{\tau\left(\varphi(X)^{-1}\right)}=\tau\left(X^{\varphi(X)^{-1}}\right)=\tau(\mathbf{d}(X))$, that is, $\tau$ commutes with both cycling and decycling.

From now on let $X \in \operatorname{USS}(X)$. It is well known that $\mathbf{c}(X) \in \operatorname{USS}(X)[18$, $\S 1$. It remains to be shown that $\tau(X)$ and $\mathbf{d}(X)$ belong to $\operatorname{USS}(X)$. Since $X \in$ $\mathrm{USS}(X) \subseteq \operatorname{SSS}(X)$, and $\tau(X)$ has the same canonical length as $X$, it follows that $\tau(X) \in \operatorname{SSS}(X)$. Moreover, one has $\mathbf{c}^{m}(X)=X$ for some $m$. Then, $\mathbf{c}^{m}(\tau(X))=$ $\tau\left(\mathbf{c}^{m}(X)\right)=\tau(X)$ and therefore $\tau(X) \in \operatorname{USS}(X)$.

Finally, $X^{X}=X \in \operatorname{USS}(X)$ and $X^{\Delta^{p+r-1}}=\tau^{p+r-1}(X) \in \operatorname{USS}(X)$. Then, by Theorem 1.13, $\mathbf{d}(X)=X^{\left(\Delta^{p} x_{1} \ldots x_{r-1}\right)}=X^{X \wedge \Delta^{p+r-1}} \in \operatorname{USS}(X)$.

Theorem 2.5. Let $X \in \mathrm{USS}(X)$ and let $s$ be a minimal simple element for $X$. Then one and only one of the following conditions holds:

(1) $\varphi(X) s$ is a simple element.

(2) $\varphi(X) s$ is left weighted as written.

Proof. Recall from Lemma 2.2 that $X^{\partial(\varphi(X))}=X^{\varphi(X)^{-1} \Delta}=(\mathbf{d}(X))^{\Delta}=\tau(\mathbf{d}(X))$. Hence, by Lemma $2.4, X^{\partial(\varphi(X))} \in \operatorname{USS}(X)$. On the other hand, since $s$ is a minimal simple element for $X$, one has $X^{s} \in \operatorname{USS}(X)$. Therefore, by Theorem 1.13 it follows that $X^{\partial(\varphi(X)) \wedge s} \in \operatorname{USS}(X)$.

Consider $t=\partial(\varphi(X)) \wedge s$. By definition, $t \preccurlyeq s$, and we just saw that $X^{t} \in$ $\operatorname{USS}(X)$. Since $s$ is a minimal simple element for $X$, this implies that either $t=s$ or $t=1$.

Notice that the first factor in the left normal form of $\varphi(X) s$ (which is possibly $\Delta$ ) is equal to $\varphi(X) s \wedge \Delta=\varphi(X) s \wedge \varphi(X) \partial(\varphi(X))=\varphi(X)(s \wedge \partial(\varphi(X)))=\varphi(X) t$. Hence it is equal to either $\varphi(X) s$ or $\varphi(X)$. The first case implies that $\varphi(X) s$ is simple, while the second one means that $\varphi(X) s$ is left weighted. 
Proposition 2.6. Let $X \in \operatorname{SSS}(X)$ with $\ell(X)>0$ and let $s$ be a simple element such that $s^{-1} X s \in \operatorname{SSS}(X)$. If $\varphi(X)$ s is left weighted, then $s \preccurlyeq \iota(X)$.

Proof. Let $\Delta^{p} x_{1} \ldots x_{r}$ be the left normal form of $X$. If $\varphi(X) s=x_{r} s$ is left weighted, then $\Delta^{p} x_{1} \ldots x_{r} s$ is the left normal form of $X s$. However, we know that $s^{-1} X s=$ $\Delta^{p} \tau^{p}(s)^{-1} x_{1} \ldots x_{r} s \in \operatorname{SSS}(X)$, hence $\tau^{p}(s)^{-1} x_{1} \ldots x_{r} s \in P$, which is equivalent to $\tau^{p}(s) \preccurlyeq x_{1} \ldots x_{r} s$. Since $\tau^{p}(s)$ is simple, this in turn is equivalent to $\tau^{p}(s) \preccurlyeq$ $x_{1} \ldots x_{r} s \wedge \Delta$. Finally, since $x_{1} \ldots x_{r} s$ is in left normal form with $r>0$, this means that $\tau^{p}(s) \preccurlyeq x_{1}$, thus $s \preccurlyeq \tau^{-p}\left(x_{1}\right)=\iota(X)$, as we wanted to show.

Corollary 2.7. Let $X \in \operatorname{USS}(X)$ with $\ell(X)>0$ and let $s$ be a minimal simple element for $X$. Then $s$ is a prefix of either $\iota(X)$ or $\iota\left(X^{-1}\right)$, or both.

Proof. We have seen in Lemma 2.1 that $\iota\left(X^{-1}\right)=\partial(\varphi(X))$ and we know by Theorem 2.5 that $\varphi(X) s$ is either simple or left weighted. In the first case, $s \preccurlyeq \partial(\varphi(X))=$ $\iota\left(X^{-1}\right)$, whereas in the second case $s \preccurlyeq \iota(X)$, by Proposition 2.6.

Notice that we could have at the same time $s \leqslant \iota(X)$ and $s \leqslant \iota\left(X^{-1}\right)$, but this is only possible in the first case, where $\varphi(X) s$ is simple.

Remark 2.8. We remark that the statements of Proposition 2.6 and Corollary 2.7 are wrong for elements $X$ with $\ell(X)=0$, as in this case $\iota(X)=\iota\left(X^{-1}\right)=1$.

Recall that every two elements in USS $(X)$ can be joined by a sequence of conjugations by minimal simple elements. We have just seen that the minimal simple elements for each $Y \in \operatorname{USS}(X)$ with $\ell(Y)>0$ are prefixes of either $\iota(Y)$ or $\iota\left(Y^{-1}\right)=\partial(\varphi(Y))$. Let us give a name to these special kinds of conjugations.

Definition 2.9. Let $X \in G$. A partial cycling of $X$ is a conjugation of $X$ by a prefix of $\iota(X)$. A partial twisted decycling of $X$ is a conjugation of $X$ by a prefix of $\iota\left(X^{-1}\right)$, or equivalently (using Lemma 2.1), by a prefix of $\partial(\varphi(X))$.

Corollary 2.10. Given $X, Y \in \mathrm{USS}(X)$, there exists a sequence of partial cyclings and partial twisted decyclings joining $X$ to $Y$.

Proof. This is a direct consequence of Theorem 1.13 and Corollary 2.7.

We end this section with several examples which will be used now and later in this paper to illustrate various points. All of our examples will be taken from braid groups, with the classical Garside structure that was discovered in [17], and with the elementary braids $\sigma_{1}, \ldots, \sigma_{n-1}$ as atoms. By Corollary 2.7, there are two kinds of minimal simple elements, hence there are two kinds of arrows in $\Gamma_{X}$. We say that an arrow $s$ starting at a vertex $Y=\Delta^{p} y_{1} \ldots y_{r}$ is black if $s$ is a prefix of $\iota(Y)$, and grey if $s$ is a prefix of $\iota\left(Y^{-1}\right)$ or, equivalently, if $y_{r} s$ is a simple element. In other words, 
an arrow starting at $Y$ is black if it corresponds to a partial cycling of $Y$, and it is grey if it corresponds to a partial twisted decycling of $Y$.

Example 2.11. Our first example is the 4-braid, $A=\sigma_{1} \sigma_{2} \sigma_{3} \sigma_{2} \sigma_{2} \sigma_{1} \sigma_{3} \sigma_{1} \sigma_{3}$. The reader is referred to Remark 1.19 and thence to $\$ 1.3$ of [3] and [22] for a brief description of the so-called Thurston-Nielsen trichotomy in mapping class groups. The braid $A$ is PA and rigid (see Remark 1.19 and Definition 1.15). Its first cycling orbit $A_{1}$ has length 3 , and by Lemma 2.4 , since $\tau\left(A_{1}\right) \neq A_{1}$ there is a second orbit $A_{2}=\tau\left(A_{1}\right)$. A computation shows that $\operatorname{USS}(A)$ has exactly these two cycling orbits, with 3 elements each, namely:

$$
\begin{aligned}
A_{1}=\left\{A_{1,1}=\right. & \sigma_{1} \sigma_{2} \sigma_{3} \sigma_{2} \cdot \sigma_{2} \sigma_{1} \sigma_{3} \cdot \sigma_{1} \sigma_{3}, \\
A_{1,2}= & \sigma_{2} \sigma_{1} \sigma_{3} \cdot \sigma_{1} \sigma_{3} \cdot \sigma_{1} \sigma_{2} \sigma_{3} \sigma_{2}, \\
A_{1,3}= & \left.\sigma_{1} \sigma_{3} \cdot \sigma_{1} \sigma_{2} \sigma_{3} \sigma_{2} \cdot \sigma_{2} \sigma_{1} \sigma_{3}\right\} \\
A_{2}=\left\{A_{2,1}=\right. & \sigma_{1} \sigma_{3} \sigma_{2} \sigma_{1} \cdot \sigma_{2} \sigma_{1} \sigma_{3} \cdot \sigma_{1} \sigma_{3}, \\
A_{2,2}= & \sigma_{2} \sigma_{1} \sigma_{3} \cdot \sigma_{1} \sigma_{3} \cdot \sigma_{1} \sigma_{3} \sigma_{2} \sigma_{1}, \\
A_{2,3}= & \left.\sigma_{1} \sigma_{3} \cdot \sigma_{1} \sigma_{3} \sigma_{2} \sigma_{1} \cdot \sigma_{2} \sigma_{1} \sigma_{3}\right\}
\end{aligned}
$$

It is easy to check (Lemma 2.4) that $\operatorname{USS}(A)$ is invariant under decycling, which for rigid braids is simply reverse cycling. Observe that $\tau\left(A_{1, j}\right)=A_{2, j}$ for $j=1,2,3$ and that $\tau^{2}=1$, so that our example also illustrates the fact that $\operatorname{USS}(A)$ is invariant under $\tau$.

The graph $\Gamma_{A}$ is illustrated in Figure 1. Since all black arrows (partial cyclings) in $\Gamma_{A}$ correspond to cyclings, the only question in constructing the graph is how to relate vertices in $A_{1}$ to vertices in $A_{2}$ by partial twisted decyclings. For that, we need to know the prefixes of the inverses. By Lemma 2.1, for every $X \in G$ one has $\iota\left(X^{-1}\right)=\partial(\varphi(X))$, and in this way we find that $\iota\left(A_{1,1}^{-1}\right)=\sigma_{2} \sigma_{1} \sigma_{3} \sigma_{2}$, and in fact
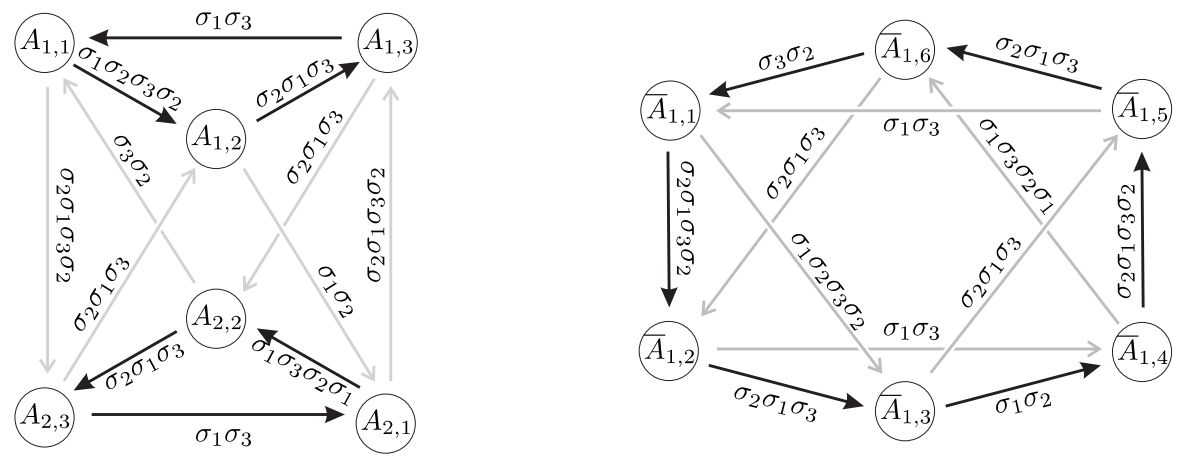

Figure 1. The graphs $\Gamma_{A}$ and $\Gamma_{\bar{A}}=\Gamma_{A^{-1}}$. 
$\left(A_{1,1}\right)^{\sigma_{2} \sigma_{1} \sigma_{3} \sigma_{2}}=A_{2,3}$. All other arrows on the graph $\Gamma_{A}$, which can be seen in the left sketch in Figure 1, can be computed in a similar way. In this very simple example it happens that both $\iota\left(A_{i, j}\right)$ and $\iota\left(A_{i, j}^{-1}\right)$ are minimal simple elements, for every $i, j$. Hence, all partial cyclings are actually cyclings, and all partial twisted decyclings are actually twisted decyclings, so they connect $A_{i, j}$ to $\tau\left(\mathbf{d}\left(A_{i, j}\right)\right)$.

Let $\bar{A}=A^{-1}$. The right sketch in Figure 1 depicts the graph $\Gamma_{A^{-1}}=\Gamma_{\bar{A}}$. There are 6 elements in the cycling orbit of $\bar{A}$. We have set $\bar{A}_{1,1}=\bar{A}$ and $\bar{A}_{1, j}=$ $\mathbf{c}^{j-1}(\bar{A}), j=2, \ldots, 6$. In this case there are 3 elements in each of 2 cycling orbits in $\Gamma_{A}$, but 6 elements in 1 cycling orbit for $\Gamma_{A^{-1}}$. We will have more to say about this and about Example $A$ (and other examples) later. See, in particular, Example 3.11 below.

In the graph $\Gamma_{A}$ all partial cyclings were actual cyclings, but that is far from the case in general:

Example 2.12. Consider the 6-braid $B=\sigma_{2} \sigma_{1} \sigma_{4} \sigma_{3} \sigma_{2} \sigma_{1} \sigma_{5} \sigma_{4} \cdot \sigma_{2} \sigma_{4}$ whose graph is depicted in Figure 2. Braid $B$, like braid $A$, is PA and rigid. A computation shows

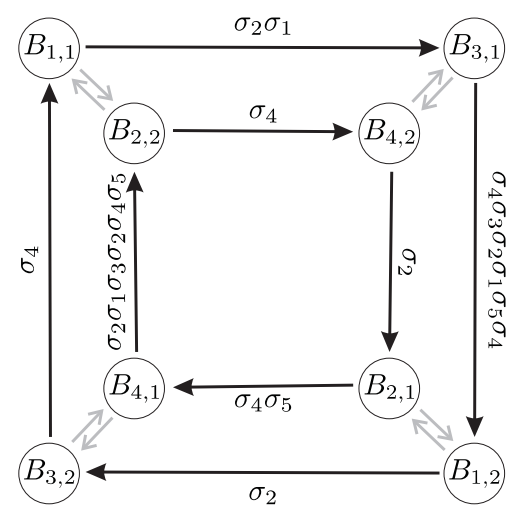

Figure 2. $\Gamma_{B}$.

that $\operatorname{USS}(B)$ has 4 cycling orbits, $B_{i}, \quad i=1,2,3,4$ with 2 elements each, $B_{i, 1}$ and $B_{i, 2}=\mathbf{c}\left(B_{i, 1}\right)=\mathbf{d}\left(B_{i, 1}\right)$. Also $\tau\left(B_{i, j}\right)=B_{i+1, j}$ for $i=1,3$ and $j=1,2$ :

$$
\begin{array}{ll}
B_{1,1}=\sigma_{2} \sigma_{1} \sigma_{4} \sigma_{3} \sigma_{2} \sigma_{1} \sigma_{5} \sigma_{4} \cdot \sigma_{2} \sigma_{4}, & B_{1,2}=\sigma_{2} \sigma_{4} \cdot \sigma_{2} \sigma_{1} \sigma_{4} \sigma_{3} \sigma_{2} \sigma_{1} \sigma_{5} \sigma_{4}, \\
B_{2,1}=\sigma_{2} \sigma_{1} \sigma_{3} \sigma_{4} \sigma_{3} \sigma_{2} \sigma_{5} \sigma_{4} \cdot \sigma_{2} \sigma_{4}, & B_{2,2}=\sigma_{2} \sigma_{4} \cdot \sigma_{2} \sigma_{1} \sigma_{3} \sigma_{4} \sigma_{3} \sigma_{2} \sigma_{5} \sigma_{4}, \\
B_{3,1}=\sigma_{1} \sigma_{4} \sigma_{3} \sigma_{2} \sigma_{1} \sigma_{5} \sigma_{4} \cdot \sigma_{2} \sigma_{1} \sigma_{4}, & B_{3,2}=\sigma_{2} \sigma_{1} \sigma_{4} \cdot \sigma_{1} \sigma_{4} \sigma_{3} \sigma_{2} \sigma_{1} \sigma_{5} \sigma_{4}, \\
B_{4,1}=\sigma_{2} \sigma_{1} \sigma_{3} \sigma_{2} \sigma_{4} \sigma_{5} \sigma_{4} \cdot \sigma_{2} \sigma_{4} \sigma_{5}, & B_{4,2}=\sigma_{2} \sigma_{4} \sigma_{5} \cdot \sigma_{2} \sigma_{1} \sigma_{3} \sigma_{2} \sigma_{4} \sigma_{5} \sigma_{4} .
\end{array}
$$

In this example every black arrow is a partial cycling (see Definition 2.9) and the concatenation of two consecutive black arrows corresponds to a cycling. Hence the 
product of four consecutive black arrows starting at $B_{i, j}$ is a circuit at $B_{i, j}$, and the product of its labels is equal to $B_{i, j}$. The grey arrow starting at $B_{i, j}$ is $\partial\left(\varphi\left(B_{i, j}\right)\right)$ for every $i, j$. Hence, it corresponds to a twisted decycling. We have not written down the labels for the grey arrows because of space considerations, but will compute some of them later, in Example 3.25.

In the graphs $\Gamma_{A}, \Gamma_{A^{-1}}$, and $\Gamma_{B}$ all arrows were either black or grey. Lest the reader think that this is always the case, the left and right sketches in Figure 3 illustrate two different types of examples. We begin with the left sketch in Figure 3:

Example 2.13. We consider the 5-braid $C=\sigma_{4} \sigma_{1} \sigma_{2} \sigma_{3} \sigma_{4}$. Since $C$ is a simple braid, we have $\inf (C)=0, \sup (C)=1, \ell(C)=1$. Its Thurston-Nielsen classification is periodic. A computer calculation shows that $\operatorname{USS}(C)$ has 12 elements, each of canonical length 1 . The graph $\Gamma_{C}$ is illustrated in the left sketch in Figure 3.
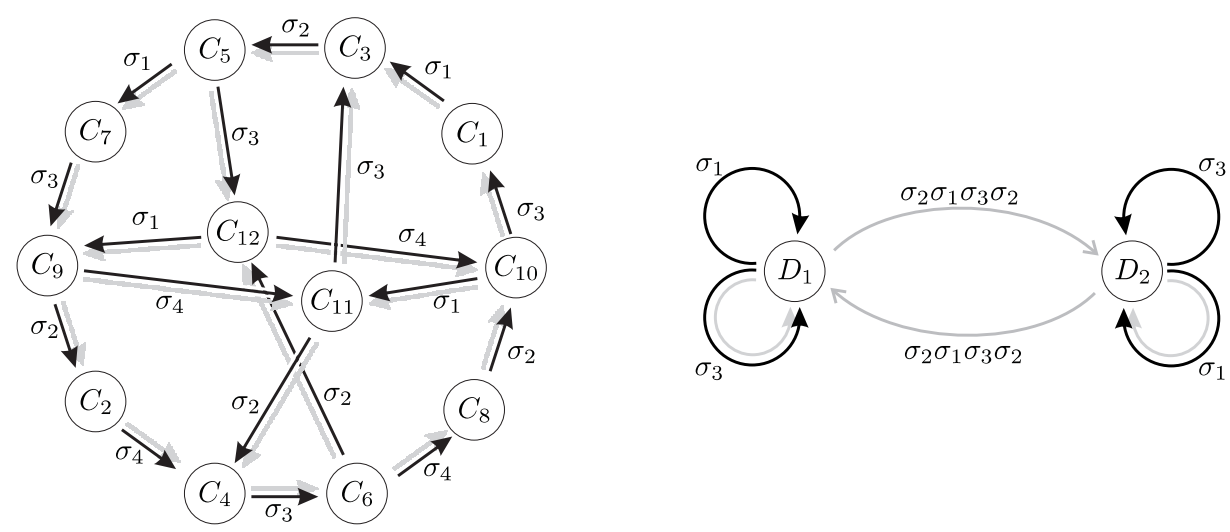

Figure $3 . \Gamma_{C}$ and $\Gamma_{D}$.

The 12 elements in $\operatorname{USS}(C)$ are labeled $C_{1}, C_{2}, \ldots, C_{12}$. We observe that, whereas in Example 2.11 every arrow was either black or grey, one sees that in Example 2.13 every arrow is both black and grey, that is, it is bi-colored! Moreover, in the braid $A$ every arrow that begins at a vertex $A_{i, j}$ is labeled by either the simple element $\iota\left(A_{i, j}\right)$ or the simple element $\iota\left(A_{i, j}^{-1}\right)$, and so is never a proper prefix, but in the braid $C$ every vertex is a simple element of letter length 5, and every arrow is labeled by a simple element of letter length 1 (an atom), from which it follows that every arrow at every vertex $C_{j}$ is a proper prefix of both $C_{j}=\iota\left(C_{j}\right)$ and of $\partial\left(C_{j}\right)=\iota\left(C_{j}^{-1}\right)$. (Note that $C_{j}$ is simple, but $C_{j}^{-1}$ is not simple.) Lest the reader think that these two extreme cases are the only ones that occur, we give one more example, in the right sketch in Figure 3. 
Example 2.14. Consider the 4-braid $D=D_{1}=\sigma_{1} \sigma_{3} \cdot \sigma_{1}$. Its graph $\Gamma_{D}$, illustrated in the right sketch in Figure 3, has arrows of all three types: black, grey and bi-colored. The Thurston-Nielsen classification of this braid shows it to be reducible. While there is no shortage of reducible braids that are rigid, this one is not rigid. Its graph has 2 cycling orbits, each having one element, the first that of $D_{1}$ and the second that of $D_{2}=\sigma_{1} \sigma_{3} \cdot \sigma_{3}$.

2.2. Black and grey components of $\operatorname{USS}(X)$. The examples that we just discussed should make it clear that we need to study and try to uncover underlying structure, if possible. Keeping in mind all of our examples, we begin our work by asking what happens to the graph $\Gamma_{X}$ if one considers only black arrows, or only grey arrows. We denote by $\Gamma_{X}^{\text {black }}\left[\Gamma_{X}^{\text {grey }}\right]$ the subgraph of $\Gamma_{X}$ having the same set of vertices, but just the black [grey] arrows. The main difference between these graphs and $\Gamma_{X}$ is that they are not necessarily connected. Therefore, in general one cannot generate the whole USS $(X)$ by using only partial cyclings [partial twisted decyclings]; both types of conjugations are, in general, required.

We denote by $\mathscr{B}_{1}, \ldots, \mathscr{B}_{S}$ the connected components of $\Gamma_{X}^{\text {black }}$ and we refer to them as the black components of $\Gamma_{X}$. In the same way, we denote by $\mathscr{g}_{1}, \ldots, \mathscr{g}_{t}$ the connected components of $\Gamma_{X}^{\text {grey }}$ and we call them grey components of $\Gamma_{X}$. Given $Y \in \operatorname{USS}(X)$, we will also denote by $\mathscr{B}_{Y}\left[\mathscr{E}_{Y}\right]$ the black [grey] component of $\Gamma_{X}$ containing $Y$ as a vertex.

In this subsection we will see that a black or grey component can be computed the same way as one computes $\Gamma_{X}$. Starting with any vertex $Y$, one can obtain any other vertex in $\mathscr{B}_{Y}\left[\mathscr{E}_{Y}\right]$ by applying repeated partial cyclings [partial twisted decyclings] to $Y$. In other words, given any two vertices $Y, Z \in \mathscr{B}_{Y}\left[\mathscr{E}_{Y}\right]$ there is a path of black [grey] arrows that goes from $Y$ to $Z$ following the sense of the arrows. In order to prove this property, we will study the paths in $\Gamma_{X}$.

Definition 2.15. An arrow in $\Gamma_{X}$ is characterized by its starting point $Y \in \operatorname{USS}(X)$ and its label $s$ which is a minimal simple element for $Y$; the endpoint then is $Y^{s}$. In order to simplify notation we denote the arrow by its label $s$. The starting point and endpoint of $s^{-1}$ are the endpoint and starting point respectively of $s$. A path in $\Gamma_{X}$ is a sequence $\left(s_{1}^{e_{1}}, \ldots, s_{k}^{e_{k}}\right)$, possibly empty, where $s_{i}$ is an arrow in $\Gamma_{X}$ and $e_{i}= \pm 1$, such that the endpoint of $s_{i}^{e_{i}}$ is equal to the starting point of $s_{i+1}^{e_{i+1}}$ for every $i=1, \ldots, k-1$. A path is black [grey], if all of its arrows are black [grey]. We say that a path $\left(s_{1}^{e_{1}}, \ldots, s_{k}^{e_{k}}\right)$ is oriented if $e_{i}=1$ for $i=1, \ldots, k$.

Notice that every path $\left(s_{1}^{e_{1}}, \ldots, s_{k}^{e_{k}}\right)$ has an associated element $\alpha=s_{1}^{e_{1}} \ldots s_{k}^{e_{k}}$ and that different paths may have the same associated element. Since the labels of arrows are simple elements, it follows that if the path is oriented then $\alpha \in P$. Finally, notice that if $X$ and $Y$ are the initial and final vertices of the above path, that is, if $X$ is the starting point of $s_{1}^{e_{1}}$ and $Y$ is the endpoint of $s_{k}^{e_{k}}$, then $X^{\alpha}=Y$. 
Notation. Given an element $Y \in G$ whose left normal form is $\Delta^{p} y_{1} \ldots y_{r}$, we define $Y^{\circ}=Y \Delta^{-p}=\tau^{-p}\left(y_{1} \ldots y_{r}\right)$.

The following result will be seen to have important consequences:

Proposition 2.16. Let $\left(s_{1}, \ldots, s_{k}\right)$ be an oriented path in $\Gamma_{X}$ starting at a vertex $Y$, and let $\alpha=s_{1} \ldots s_{k} \in P$ be its corresponding element. Then one has:

(1) If $\alpha \preccurlyeq Y^{\circ}$ then $\left(s_{1}, \ldots, s_{k}\right)$ is an oriented black path.

(2) If $\alpha \preccurlyeq\left(Y^{-1}\right)^{\circ}$ then $\left(s_{1}, \ldots, s_{k}\right)$ is an oriented grey path.

Proof. The claim is trivial if $\alpha=1$, so we may assume that $\alpha \neq 1$. We will show the result by induction on $k$. Suppose that $\alpha \preccurlyeq Y^{\circ}$. Notice that $\inf \left(Y^{\circ}\right)=0$ and $\iota\left(Y^{\circ}\right)=\tau^{-p}\left(y_{1}\right)=\iota(Y)$. Hence, since $\alpha \preccurlyeq Y^{\circ}$ it follows that $\inf (\alpha)=0$ and then $s_{1} \preccurlyeq \iota(\alpha) \preccurlyeq \iota\left(Y^{\circ}\right)=\iota(Y)$, that is, $s_{1}$ is a black arrow. In particular, the result is true if $k=1$. Suppose that $k>1$ and that the result is true for oriented paths of length $k-1$.

We already know that $s_{1}$ is a black arrow. Now denote $T=Y^{s_{1}}$ and notice that one has $T^{\circ}=s_{1}^{-1} Y^{\circ} \tau^{-p}\left(s_{1}\right)$ since $T \in \operatorname{USS}(X) \subseteq \operatorname{SSS}(X)$. Hence, since $\alpha=s_{1} \ldots s_{k} \preccurlyeq Y^{\circ}$ it follows that $s_{2} \ldots s_{k} \preccurlyeq s_{1}^{-1} Y^{\circ} \preccurlyeq T^{\circ}$. By induction hypothesis $\left(s_{2}, \ldots, s_{k}\right)$ is an oriented black path, hence $\left(s_{1}, \ldots, s_{k}\right)$ is also a black path and the result follows.

The proof for $s \preccurlyeq\left(Y^{-1}\right)^{\circ}$ is similar; for the induction step note that Remark $1.8 \mathrm{im}$ plies $T^{-1} \in \operatorname{SSS}\left(X^{-1}\right)$ for any $T \in \operatorname{USS}(X)$, whence $\left(T^{-1}\right)^{\circ}=s_{1}^{-1}\left(Y^{-1}\right)^{\circ} \tau^{-p}\left(s_{1}\right)$ if $T=Y^{s_{1}}$.

The following is a particular case of the above result, which makes the connection with partial cyclings and partial twisted decyclings.

Corollary 2.17. Let $\left(s_{1}, \ldots, s_{k}\right)$ be an oriented path in $\Gamma_{X}$ starting at a vertex $Y$. If the associated element $s=s_{1} \ldots s_{k}$ is simple, then one has:

(1) If $s \preccurlyeq \iota(Y)$ then $\left(s_{1}, \ldots, s_{k}\right)$ is an oriented black path.

(2) If $s \preccurlyeq \iota\left(Y^{-1}\right)$ then $\left(s_{1}, \ldots, s_{k}\right)$ is an oriented grey path.

Proof. If $s$ is simple, then $s \preccurlyeq Y^{\circ}$ if and only if $s \preccurlyeq \iota\left(Y^{\circ}\right)=\iota(Y)$, and $s \preccurlyeq\left(Y^{-1}\right)^{\circ}$ if and only if $s \preccurlyeq \iota\left(\left(Y^{-1}\right)^{\circ}\right)=\iota\left(Y^{-1}\right)$. Therefore, this result is a direct consequence of Proposition 2.16.

Example 2.18. The converse of the above result is not true. For instance, consider the braid $U=\sigma_{1} \sigma_{2} \sigma_{1} \sigma_{3} \sigma_{2}$, a simple element in $B_{4}$. Then $\left(\sigma_{1}, \sigma_{3}\right)$ is a black path in $\Gamma_{U}$ starting at $U$, also $s=\sigma_{1} \sigma_{3}$ is simple, but $s$ is not a prefix of $\iota(U)=U$. Moreover $\left(\sigma_{1}, \sigma_{3}\right)$ is also a grey path but $\left(\sigma_{1} \sigma_{2} \sigma_{1} \sigma_{3} \sigma_{2}\right)\left(\sigma_{1} \sigma_{3}\right)$ is not simple, thus $s \nless \iota\left(U^{-1}\right)$. Hence this is a counterexample for the converse of the two properties above. 
Proposition 2.19. Given $Y \in \mathrm{USS}(X)$ and a black [grey] arrow $s$ in $\Gamma_{X}$ starting at $Y$, there exists an oriented black $[$ grey $]$ path $\left(s_{1}, \ldots, s_{k}\right)$ in $\Gamma_{X}$ starting and ending at $Y$, such that $s_{1}=s$.

Proof. Suppose that $s$ is a black arrow and let $p=\inf (Y)$. Since $Y^{Y^{\circ}}=\tau^{-p}(Y) \in$ $\operatorname{USS}(X)$, we know by Proposition 2.16 that every decomposition of $Y^{\circ}$ as a product of minimal simple elements corresponds to an oriented black path. Moreover, $s$ is a black arrow, that is, $s \preccurlyeq \iota(Y) \preccurlyeq Y^{\circ}$, whence there is a decomposition of $Y^{\circ}$ as a product of minimal simple elements whose first factor is $s$. Therefore, there exists a black path $\left(s_{1}, \ldots, s_{t}\right)$ in $\Gamma_{X}$, going from $Y$ to $\tau^{-p}(Y)$, such that $s_{1}=s$.

We can apply the same reasoning to show that there is an oriented black path from $\tau^{-(m-1) p}(Y)$ to $\tau^{-m p}(Y)$ for every $m \geq 1$. Concatenating these paths we obtain for every $m \geq 1$ an oriented black path from $Y$ to $\tau^{-m p}(Y)$ whose first arrow is $s$. Since $\Delta^{e}$ is central for some integer $e$, it follows that for $m=e$ there is a black path $\left(s_{1}, \ldots, s_{k}\right)$ going from $Y$ to $\tau^{-e p}(Y)=Y$, such that $s_{1}=s$.

The analogous proof works for grey arrows. We just need to notice that $Y^{\left(Y^{-1}\right)^{\circ}}=$ $\left(\left(Y^{-1}\right)^{\left(Y^{-1}\right)^{\circ}}\right)^{-1}=\left(\tau^{p+r}\left(Y^{-1}\right)\right)^{-1}=\tau^{p+r}(Y) \in \operatorname{USS}(X)$, where $r=\ell(Y)$.

Corollary 2.20. Given two elements $Y$ and $Z$ in a black component $\mathscr{B}_{i}$ [grey component $\mathscr{E}_{i}$ ] of $\Gamma_{X}$, there exists an oriented black [grey] path going from $Y$ to $Z$.

Proof. Suppose that $Y$ and $Z$ belong to the same black component. Hence one can go from $Y$ to $Z$ along a black path $\left(s_{1}^{e_{1}}, \ldots, s_{t}^{e_{t}}\right)$. Suppose that $e_{j}=-1$ for some $j$, where $s_{j}$ is a black arrow going from $U$ to $V$ (so $s_{j}^{-1}$ goes from $V$ to $U$ ). By the above result there exists an oriented black path $\left(s_{j}, b_{2}, \ldots, b_{k}\right)$ going from $U$ to itself. Therefore $\left(b_{2}, \ldots, b_{k}\right)$ is an oriented black path going from $V$ to $U$, so one can replace $s_{j}^{-1}$ by $\left(b_{2}, \ldots, b_{k}\right)$ in the above path. Applying the same procedure for every $j$ such that $e_{j}=-1$, we obtain an oriented black path going from $Y$ to $Z$. The analogous proof works for grey arrows.

We establish one more property of the black and grey components:

Proposition 2.21. The set of vertices in a black component of $\Gamma_{X}$ is a union of orbits under cycling. This is not necessarily true for grey components.

Proof. To prove the first statement, we just need to show that $\mathbf{c}(Y)$ is a vertex of $\mathcal{B}_{Y}$, for every $Y \in \operatorname{USS}(X)$. But this is clear, since $\iota(Y)$ can be decomposed as a product of minimal simple elements, $\iota(Y)=s_{1} \ldots s_{k}$. By Corollary 2.17, the path $\left(s_{1}, \ldots, s_{k}\right)$ is black, and it goes from $Y$ to $Y^{\imath(Y)}=\mathbf{c}(Y)$. Hence, $Y$ and $\mathbf{c}(Y)$ belong to the same black component, as we wanted to show.

To prove that the analogous statement for grey components is false, it suffices to produce an example. See the graph $\Gamma_{B}$ in Figure 2. Observe that $\left\{B_{1,1}, B_{2,2}\right\}$ is not a union of orbits. 
Thanks to the above results, we can give an algorithm to compute the black or the grey component of an element in its USS. It is analogous to the corresponding algorithms computing $\operatorname{SSS}(X)$ [14], [16] or $\operatorname{USS}(X)$ [18]. We just need to recall from Theorem 1.13 that given $Y \in \operatorname{USS}(X)$ and an atom $a \in P$, there exists a unique element $c_{Y}(a)$ minimal with respect to the condition that $Y^{c_{Y}(a)} \in \operatorname{USS}(Y)$ and $a \preccurlyeq c_{Y}(a)$.

The algorithm to compute $\mathcal{B}_{X}$ goes as follows. Starting with $X$, conjugate it by all its minimal simple elements which are prefixes of $\iota(X)$. For each new element $Y$ which appears in this way, conjugate it by all its minimal simple elements which are prefixes of $\iota(Y)$. Keep going until no new element appears. At this point, by Corollary 2.20, the black component $\mathscr{B}_{X}$ has been computed. The computation of $\mathscr{E}_{X}$ is analogous. More precisely, the algorithms are as follows.

\section{Algorithm 1}

Input: $X \in \operatorname{USS}(X)$.

Output: $\mathscr{B}_{X}$.

(1) Set $\mathcal{V}=\{X\}$ and $\mathcal{V}^{\prime}=\emptyset$.

(2) While $\mathcal{V} \neq \mathcal{V}^{\prime}$ do

(a) Take $Y \in \mathcal{V} \backslash \mathcal{V}^{\prime}$.

(b) For every atom $a \preccurlyeq \iota(Y)$ do

(i) Compute $c_{Y}(a)$.

(ii) If $c_{Y}(a)$ is a minimal simple element, set $\mathcal{V}=\mathcal{V} \cup\left\{Y^{c_{Y}(a)}\right\}$, and store $c_{Y}(a)$ as a black arrow going from $Y$ to $Y^{c_{Y}(a)}$.

(c) Set $\mathcal{V}^{\prime}=\mathcal{V}^{\prime} \cup\{Y\}$.

(3) Return $\mathcal{V}$, together with the information on all black arrows.

\section{Algorithm 2}

Input: $X \in \operatorname{USS}(X)$.

Output: $\mathscr{G}_{X}$.

(1) Set $\mathcal{V}=\{X\}$ and $\mathcal{V}^{\prime}=\emptyset$.

(2) While $\mathcal{V} \neq \mathcal{V}^{\prime}$ do

(a) Take $Y \in \mathcal{V} \backslash \mathcal{V}^{\prime}$.

(b) For every atom $a$ such that $\varphi(Y) a$ is simple do

(i) Compute $c_{Y}(a)$.

(ii) If $c_{Y}(a)$ is a minimal simple element, set $\mathcal{V}=\mathcal{V} \cup\left\{Y^{c_{Y}(a)}\right\}$, and store $c_{Y}(a)$ as a grey arrow going from $Y$ to $Y^{c_{Y}(a)}$.

(c) Set $\mathcal{V}^{\prime}=\mathcal{V}^{\prime} \cup\{Y\}$.

(3) Return $\mathcal{V}$, together with the information on all grey arrows. 
2.3. Intersection of black and grey components. Now that we know how to compute the black and grey components of any element in $\operatorname{USS}(X)$, we will see how this can be used to solve the conjugacy problem in $G$. Recall that the algorithm in [18] is based on the fact that $X \in \operatorname{USS}(X)$ and $Y \in \operatorname{USS}(Y)$ are conjugate if and only if $\operatorname{USS}(X)=\operatorname{USS}(Y)$, or equivalently, if $Y \in \operatorname{USS}(X)$. But the analogous statement does not hold for black or grey components, since $\Gamma_{X}^{\text {black }}$ and $\Gamma_{X}^{\text {grey }}$ are not necessarily connected. That is, we could have two conjugate elements $X, Y \in \operatorname{USS}(X)$ such that $\mathscr{B}_{X} \cap \mathscr{B}_{Y}=\emptyset$. Hence, computing just black components (or just grey components) will not solve the conjugacy problem, in general.

However, we will see in this section that it is actually possible to determine whether $X \in \operatorname{USS}(X)$ and $Y \in \operatorname{USS}(Y)$ are conjugate just by computing $\mathscr{B}_{X}$ and $\mathscr{E}_{Y}$, since every black component intersects every grey component of $\Gamma_{X}$. Hence, $X$ and $Y$ are conjugate if and only if $\mathcal{B}_{X} \cap \mathscr{G}_{Y} \neq \emptyset$. This result will be achieved by showing that every two elements in USS $(X)$ can be joined by a grey path followed by a black path. First we need two preliminary results.

Proposition 2.22. Let $\left(s_{1}, \ldots, s_{k}\right)$ be an oriented path in $\Gamma_{X}$ starting at a vertex $Y$, with $\ell(Y)>0$. If the associated element $s=s_{1} \ldots s_{k}$ is simple, then one has:

(1) If $\varphi(Y) s$ is left weighted then $\left(s_{1}, \ldots, s_{k}\right)$ is an oriented black path.

(2) If $\varphi\left(Y^{-1}\right) s$ is left weighted then $\left(s_{1}, \ldots, s_{k}\right)$ is an oriented grey path.

Proof. The first claim follows immediately from Proposition 2.6 and Corollary 2.17. The second claim is proved by applying the same techniques to $Y^{-1} \in \operatorname{SSS}\left(X^{-1}\right)$.

Let us extend the above result to non-simple elements.

Proposition 2.23. Let $X \in G$ and $Y \in \operatorname{USS}(X)$, with $\ell(Y)>0$. Suppose that $\alpha \in P$ is such that $\inf (\alpha)=0$ and $Y^{\alpha} \in \operatorname{USS}(X)$.

If $\varphi(Y) \iota(\alpha)$ is left weighted, then $\alpha$ can be decomposed as $\alpha=s_{1} \ldots s_{k}$, where $\left(s_{1}, \ldots, s_{k}\right)$ is an oriented black path.

If $\varphi\left(Y^{-1}\right) \iota(\alpha)$ is left weighted, then $\alpha$ can be decomposed as $\alpha=s_{1} \ldots s_{k}$, where $\left(s_{1}, \ldots, s_{k}\right)$ is an oriented grey path.

Proof. If $\alpha=1$ the result follows from Proposition 2.22. Let $\Delta^{p} y_{1} \ldots y_{r}$ be the left normal form of $Y$ and let $\alpha_{1} \ldots \alpha_{t}$ be the left normal form of $\alpha$. Since $\alpha_{1}=\alpha \wedge \Delta$, Theorem 1.13 tells us that $Y^{\alpha_{1}} \in \operatorname{USS}(X)$. Suppose first that $\varphi(Y) \iota(\alpha)=y_{r} \alpha_{1}$ is left weighted. Then, by Proposition 2.22, $\alpha_{1}$ can be decomposed as a product of black arrows.

Now consider $Z=Y^{\alpha_{1}} \in \operatorname{USS}(X) \subseteq \operatorname{SSS}(X)$ whose left normal form is $\Delta^{p} z_{1} \ldots z_{r}$, and $\alpha^{\prime}=\alpha_{2} \ldots \alpha_{t}$. The result will follow by induction on $t$ if we show that $z_{r} \alpha_{2}$ is left weighted. But since $y_{r} \alpha_{1}$ is left weighted and $Z=\alpha_{1}^{-1} \Delta^{p} y_{1} \ldots y_{r} \alpha_{1}$, it follows (see, for example, [18, Proposition 2.1]) that $z_{r}=\beta \alpha_{1}$, where $y_{r} \succcurlyeq \beta$. 
Therefore $z_{r} \alpha_{2}=\left(\beta \alpha_{1}\right) \alpha_{2}$ is left weighted, since so is $\alpha_{1} \alpha_{2}$. Hence, $\varphi(Z) \iota\left(\alpha^{\prime}\right)=$ $z_{r} \alpha_{2}$ is left weighted, so induction on $t$ can be applied and the result follows.

If $\varphi\left(Y^{-1}\right) \alpha$ is left weighted, we can apply a similar reasoning to show that $\alpha=$ $s_{1} \ldots s_{k}$ where $\left(s_{1}, \ldots, s_{k}\right)$ is an oriented grey path. For the induction step note that if $Z=Y^{\alpha_{1}} \in \operatorname{USS}(X)$, then $Z^{-1}=\left(Y^{-1}\right)^{\alpha_{1}} \in \operatorname{SSS}\left(X^{-1}\right)$.

Now we are able to show that every pair of elements in $\operatorname{USS}(X)$ can be joined by a (possibly empty) grey path followed by a (possibly empty) black path, and also by a (possibly empty) black path followed by a (possibly empty) grey path. The blackgrey path and the grey-black path can either be distinct or coincide. For examples, see Figure 2.

Theorem 2.24. Let $X \in G$. Given $Y, Z \in \operatorname{USS}(X)$, with $\ell(Y)>0$, there exists an oriented path $\left(g_{1}, \ldots, g_{s}, b_{1}, \ldots, b_{t}\right)$ in $\Gamma_{X}$ going from $Y$ to $Z$, such that $\left(g_{1}, \ldots, g_{s}\right)$ is a (possibly empty) grey path and $\left(b_{1}, \ldots, b_{t}\right)$ is a (possibly empty) black path. If $Z=Y^{\alpha}$ with $\alpha \in P$, then the paths can be chosen such that $g_{1} \ldots g_{s} b_{1} \ldots b_{t}=\alpha$.

Proof. Since $Y$ and $Z$ are conjugate, there exists some $\alpha \in G$ such that $Y^{\alpha}=Z$. Let $\Delta^{m} \alpha_{1} \ldots \alpha_{t}$ be the left normal form of $\alpha$. Since some power of $\Delta$, say $\Delta^{e}$, belongs to the center of $G$, it follows that $\Delta^{k e+m} \alpha_{1} \ldots \alpha_{t}$ also conjugates $Y$ to $Z$ for every integer $k$. Hence we can assume $\inf (\alpha) \geq 0$, that is, $\alpha \in P$.

Denote $Y^{(1)}=Y$, and let $\Delta^{p} y_{1} \ldots y_{r}$ be its left normal form. Moreover let $\alpha^{(1)}=\alpha$. If $\inf \left(\alpha^{(1)}\right)>0$ then $\Delta \preccurlyeq \alpha^{(1)}$, so every simple element is a prefix of $\alpha^{(1)}$. In particular, all grey arrows for $Y^{(1)}$ are prefixes of $\alpha^{(1)}$. Let us then choose $g_{1} \preccurlyeq \partial\left(y_{r}\right) \preccurlyeq \alpha^{(1)}$, a grey arrow starting at $Y^{(1)}$, and denote $Y^{(2)}=Y^{g_{1}}$ and $\alpha^{(2)}=g_{1}^{-1} \alpha^{(1)}$. We continue this process while $\inf \left(\alpha^{(i)}\right)>0$, finding grey arrows $g_{1}, \ldots, g_{i}$ such that $\alpha=g_{1} \ldots g_{i} \alpha^{(i+1)}$ and $Y^{(i+1)}=Y^{g_{1} \ldots g_{i}}$. Since the length of possible decompositions of $\alpha$ as a product of simple elements is finite, this process must stop, and we will have $\alpha=g_{1} \ldots g_{k-1} \alpha^{(k)}$, where $\left(g_{1}, \ldots, g_{k-1}\right)$ is an oriented grey path and $\inf \left(\alpha^{(k)}\right)=0$. Notice that $\alpha^{(k)}$ conjugates $Y^{(k)}=Y^{g_{1} \ldots g_{k-1}}$ to $Z$.

Now let $\Delta^{p} y_{1}^{(k)} \ldots y_{r}^{(k)}$ be the left normal form of $Y^{(k)}$, and suppose that $y_{r}^{(k)} \iota\left(\alpha^{(k)}\right)$ is not left weighted. This means that $\partial\left(y_{r}^{(k)}\right) \wedge \iota\left(\alpha^{(k)}\right) \neq 1$. By Theorem 1.13, this element conjugates $Y^{(k)}$ to another element in $\operatorname{USS}(X)$, hence there is a minimal simple element $g_{k} \preccurlyeq \partial\left(y_{r}^{(k)}\right) \wedge \iota\left(\alpha^{(k)}\right)$. Since $g_{k} \preccurlyeq \partial\left(y_{r}^{(k)}\right)$, one has that $g_{k}$ is a grey arrow and since $g_{k} \preccurlyeq \iota\left(\alpha^{(k)}\right)$, it follows that $\alpha^{(k+1)}=g_{k}^{-1} \alpha^{(k)} \in P$. We can continue this process while $\varphi\left(Y^{(i)}\right) \iota\left(\alpha^{(i)}\right)$ is not left weighted, adding new arrows to our oriented grey path dividing $\alpha$.

As above, this process must stop. We will then obtain $\alpha=g_{1} \ldots g_{s} \alpha^{(s+1)}$, and $Y^{(s+1)}=Y^{g_{1} \ldots g_{s}} \in \operatorname{USS}(X)$, where $\left(g_{1}, \ldots, g_{s}\right)$ is a grey path, $\inf \left(\alpha^{(s+1)}\right)=0$ and $\varphi\left(Y^{(s+1)}\right) \iota\left(\alpha^{(s+1)}\right)$ is left weighted. Since $\alpha^{(s+1)}$ conjugates $Y^{(s+1)}$ to $Z \in \operatorname{USS}(X)$, it follows by Proposition 2.23 that $\alpha^{(s+1)}$ can be decomposed as a product of black arrows, as we wanted to show. 
Theorem 2.25. Let $X \in G$. Given $Y, Z \in \operatorname{USS}(X)$, with $\ell(Y)>0$, there exists an oriented path $\left(b_{1}, \ldots, b_{t}, g_{1}, \ldots, g_{s}\right)$ in $\Gamma_{X}$ going from $Y$ to $Z$, such that $\left(b_{1}, \ldots, b_{t}\right)$ is a (possibly empty) black path and $\left(g_{1}, \ldots, g_{s}\right)$ is a (possibly empty) grey path. If $Z=Y^{\alpha}$ with $\alpha \in P$, then the paths can be chosen such that $b_{1} \ldots b_{t} g_{1} \ldots g_{s}=\alpha$.

Proof. The proof is analogous to the proof of the previous result. There exists some $\alpha \in P$ such that $Y^{\alpha}=Z$. We then construct a sequence $\left\{Y^{(i)}\right\}_{i \geq 1}$ and $\left\{\alpha^{(i)}\right\}_{i \geq 1}$, where $\alpha^{(1)}=\alpha$ and $Y^{(1)}=Y$. If inf $\left(\alpha^{(i)}\right)>0$, there exists a black arrow $b_{i}$ dividing $\alpha^{(i)}$, and we define $Y^{(i+1)}=\left(Y^{(i)}\right)^{b_{i}}$ and $\alpha^{(i+1)}=b_{i}^{-1} \alpha^{(i)}$.

If $\inf \left(\alpha^{(i)}\right)=0$ and $\varphi\left(\left(Y^{(i)}\right)^{-1}\right) \iota\left(\alpha^{(i)}\right)$ is not left weighted, then there is a prefix $\beta \preccurlyeq \iota\left(\alpha^{(i)}\right)$ such that $\varphi\left(\left(Y^{(i)}\right)^{-1}\right) \beta$ is simple and $\left(Y^{(i)}\right)^{\beta} \in \operatorname{USS}(X)$, for instance, one can take $\beta=\partial\left(\varphi\left(\left(Y^{(i)}\right)^{-1}\right)\right) \wedge \iota\left(\alpha^{(i)}\right) \preccurlyeq \partial\left(\varphi\left(\left(Y^{(i)}\right)^{-1}\right)\right)=\iota\left(Y^{(i)}\right)$ by Lemma 2.1. Hence, every minimal simple element dividing $\beta$ (and thus $\alpha^{(i)}$ ) is a black arrow. Therefore, if $\varphi\left(\left(Y^{(i)}\right)^{-1}\right) \iota\left(\alpha^{(i)}\right)$ is not left weighted, there exists a black arrow dividing $\alpha^{(i)}$.

We keep going, finding new black arrows dividing each $\alpha^{(j)}$, until we obtain some $\alpha^{(t+1)}$ such that $\varphi\left(\left(Y^{(t+1)}\right)^{-1}\right) \iota\left(\alpha^{(t+1)}\right)$ is left weighted. Then, by Proposition 2.23, $\alpha^{(t+1)}$ can be decomposed as a product of grey arrows, and the result follows.

Corollary 2.26. Let $X \in G$. Given $Y, Z \in \operatorname{USS}(X)$, one has $\mathcal{B}_{Y} \cap \mathscr{E}_{Z} \neq \emptyset$.

Proof. This is a straightforward consequence of the previous result. For instance, we know that there exists an oriented path $\left(b_{1}, \ldots, b_{t}, g_{1}, \ldots, g_{s}\right)$ going from $Y$ to $Z$, such that $\left(b_{1}, \ldots, b_{t}\right)$ is a black path and $\left(g_{1}, \ldots, g_{s}\right)$ is a grey path. If we define $V=Y^{b_{1} \ldots b_{t}}=Z^{g_{s}^{-1} \ldots g_{1}^{-1}}$, then $V$ belongs to the same black component as $Y$, and to the same grey component as $Z$, hence $V \in \mathscr{B}_{Y} \cap \mathscr{E}_{Z}$.

Corollary 2.27. Given $X, Y \in G$, let $X^{\prime} \in \operatorname{USS}(X)$ and $Y^{\prime} \in \operatorname{USS}(Y)$. Then $X$ and $Y$ are conjugate if and only if $\mathscr{B}_{X^{\prime}} \cap \mathcal{E}_{Y^{\prime}} \neq \emptyset$.

Proof. We know that $X$ and $Y$ are conjugate if and only if $\operatorname{USS}(X)=\operatorname{USS}(Y)$. In this case, $X^{\prime}, Y^{\prime} \in \operatorname{USS}(X)$, hence Corollary 2.26 tells us that $\mathscr{B}_{X^{\prime}} \cap \mathscr{E}_{Y^{\prime}} \neq \emptyset$. Conversely, if there exists some $V \in \mathscr{B}_{X^{\prime}} \cap \mathscr{E}_{Y^{\prime}}$, then $V$ is conjugate to $X^{\prime}$ and also to $Y^{\prime}$. Since $X$ is conjugate to $X^{\prime}$ and $Y$ is conjugate to $Y^{\prime}$, it follows that $X$ and $Y$ are conjugate.

Using the above results, we will be able to obtain a new algorithm to solve the CDP/CSP in Garside groups. See $\$ 5.2$ below. 


\section{Rigid elements}

Rigid elements in Garside groups were studied in [3]. The structure of $\Gamma_{X}$ which we described in the previous section, will be seen in this section to be particularly simple in the case of rigid elements. This is important for the following reasons:

(1) It was proved in Theorems 3.21 and 3.22 of [3] that a wide class of elements in Garside groups have rigid powers. Also, if $X$ has a rigid power, then all elements in $\operatorname{USS}(X)$ have rigid powers. Moreover if one element in $\operatorname{USS}\left(X^{k}\right)$ is rigid, then all elements in $\operatorname{USS}\left(X^{k}\right)$ are rigid.

(2) Corollary 3.24 of [3] asserts, in particular, that every PA braid has a small power whose ultra summit set consists of rigid braids, also an explicit bound for the power was found in $§ 3.5$ of [3]. This allows us to study the conjugacy problem for PA braids, using the properties of rigid elements.

As noted earlier, the ultra summit set of a rigid element in a Garside group often exhibits a transparently simple structure similar to the one seen in Example 2.11, that is, the cycling orbits have length $\ell(X)$ or $2 \ell(X)$ and the number of orbits is 2 or 1 . However, there are examples of braids (and thus of elements in Garside groups) whose ultra summit set is bigger than expected. Hence, a deeper study of ultra summit sets is needed to understand this phenomenon. As will be seen, the combinatorics are quite complicated and the bounds we seek are well-hidden.

3.1. Vertices and arrows in the USS graph of a rigid element. The effect of a partial cycling on the normal form of a rigid element can be described explicitly:

Lemma 3.1. Let $X \in G$ be a rigid element, whose left normal form is $\Delta^{p} x_{1} \ldots x_{r}$. Let $s \leqslant \iota(X)$, such that $X^{s} \in \operatorname{SSS}(X)$. Then there is a decomposition of each factor $x_{i}=a_{i} b_{i}$, such that $a_{i}, b_{i}$ are simple elements for $i=1, \ldots, r$, also $s=\tau^{-p}\left(a_{1}\right)$, and the left normal forms of $X$ and $X^{s}$ are

$$
X=\Delta^{p}\left(a_{1} b_{1}\right)\left(a_{2} b_{2}\right) \ldots\left(a_{r} b_{r}\right), \quad X^{s}=\Delta^{p}\left(b_{1} a_{2}\right)\left(b_{2} a_{3}\right) \ldots\left(b_{r} \tau^{-p}\left(a_{1}\right)\right) .
$$

Proof. As $s \preccurlyeq \iota(X)=\tau^{-p}\left(x_{1}\right)$, we have $a_{1} \preccurlyeq x_{1}$, where $a_{1}=\tau^{p}(s)$. Moreover, by the rigidity of $X$, the left normal form of $X^{\tau^{-p}\left(x_{1}\right)}$ is $\Delta^{p} x_{2} \ldots x_{r} \tau^{-p}\left(x_{1}\right)=$ $\Delta^{p}\left(x_{1}^{-1} x_{1} x_{2}\right) \ldots\left(x_{r-1}^{-1} x_{r-1} x_{r}\right)\left(x_{r}^{-1} x_{r} \tau^{-p}\left(x_{1}\right)\right)$. Since $X^{s} \in \operatorname{SSS}(X)$, the left normal form of $X^{s}$ is given by $\Delta^{p}\left(a_{1}^{-1} x_{1} a_{2}\right) \ldots\left(a_{r-1}^{-1} x_{r-1} a_{r}\right)\left(a_{r}^{-1} x_{r} \tau^{-p}\left(a_{1}\right)\right)$, where $a_{i} \preccurlyeq x_{i}$ for $i=1, \ldots, r$ by monotonicity [18, Corollary 2.2]. Defining $b_{i}=a_{i}^{-1} x_{i}$ for $i=1, \ldots, r$ shows the claim.

We remark that the above result also follows easily from [8, Proposition 3.1] or [21, Lemma 4.6].

Concerning the arrows of $\Gamma_{X}$, the first difference between the case of a rigid element and the general case is the following: 
Lemma 3.2. Let $X \in \operatorname{USS}(X)$ and $\ell(X)>0$. Then $X$ is rigid if and only if there are no bi-colored arrows starting at $X$.

Proof. If an arrow $s$ starting at $X$ is black and grey at the same time, then it is a left divisor of both $\iota(X)$ and $\iota\left(X^{-1}\right)$. This is only possible if $\iota(X) \wedge \iota\left(X^{-1}\right) \neq 1$, that is, if $X$ is not rigid.

Conversely, suppose that $X$ is not rigid. We know that $X^{\iota(X)}=\mathbf{c}(X) \in \operatorname{USS}(X)$, but also $X^{\iota\left(X^{-1}\right)}=\tau(\mathbf{d}(X)) \in \operatorname{USS}(X)$. Hence, by Theorem $1.13, X^{\iota(X) \wedge \iota\left(X^{-1}\right)} \in$ $\operatorname{USS}(X)$, so there is a minimal simple element for $X$ which is a divisor of $\iota(X) \wedge$ $\iota\left(X^{-1}\right)$. In other words, there is a bi-colored arrow starting at $X$.

We now recall several results about the vertices in $\operatorname{USS}(X)$, when $X$ is rigid, all proved by the authors of this paper in the manuscript [3].

Proposition 3.3 ([3, Theorem 3.15]). Let $X$ be rigid and $\ell(X)>1$. Then every element in the ultra summit set of $X$ is rigid.

Corollary 3.4 ([3, Corollary 3.16]). If $X$ is rigid and $\ell(X)>1$, then $\operatorname{USS}(X)$ is the set of rigid conjugates of $X$.

Corollary 3.5 ([3, Corollary 3.17]). If $X$ is rigid and $\ell(X)>1$, then $\operatorname{USS}\left(X^{-1}\right)$ is the set of inverses of the elements in $\operatorname{USS}(X)$.

Corollary 3.6. Let $X \in \operatorname{USS}(X)$ and $\ell(X)>1$. Then $X$ is rigid if and only if there are no bi-colored arrows in $\Gamma_{X}$.

Proof. This follows directly from Lemma 3.2 and Proposition 3.3.

For example, the reader may wish to go back and compare Example 2.11 and Example 2.12, both of which were rigid braids, and Example 2.13 and Example 2.14, which were not rigid braids. In this regard, we note that Example 2.13 is a periodic braid (peek ahead to $\S 4$ ), whereas Example 2.14 is reducible. The additional structure in the periodic case will be elucidated when we get to $\$ 4$.

We continue our investigations of the graph of $\operatorname{USS}(X)$ when $X$ is rigid. We know that $X$ is rigid if and only if $X^{-1}$ is rigid. Now we show that the arrows starting at $X$ in $\Gamma_{X}$ are the same as the arrows starting at $X^{-1}$ in $\Gamma_{X^{-1}}$.

The next lemma will be important when we study the periodic elements in the braid groups, in [4], We will also use it in the proof of Proposition 3.8.

Lemma 3.7. If $X \in \operatorname{SSS}(X)$ and $\ell(X)=1$, then $X \in \operatorname{USS}(X)$. Moreover, $\operatorname{USS}(X)=\operatorname{SSS}(X)$, and it consists of the conjugates to $X$ whose canonical length is 1 . 
Proof. Notice that, if $\ell(X)=1$ then $\mathbf{c}(X)=\tau^{-p}(X)$, where $p=\inf (X)$. Since some power of $\tau$ is trivial, it follows that $\mathbf{c}^{k}(X)=X$ for some $k$. Therefore, $X \in \operatorname{USS}(X)$.

If we apply this reasoning to every element in $\operatorname{SSS}(X)$, it follows that $\operatorname{SSS}(X) \subseteq$ $\operatorname{USS}(X)$. Since $\operatorname{USS}(X) \subseteq \operatorname{SSS}(X)$ by definition, equality holds. By definition, $\operatorname{SSS}(X)$ consists of the conjugates to $X$ of minimal canonical length. Since $X \in$ $\operatorname{SSS}(X)$, and $\ell(X)=1$, the elements in $\operatorname{SSS}(X)=\operatorname{USS}(X)$ are precisely the conjugates of $X$ of canonical length 1 .

Proposition 3.8. Let $X$ be rigid. Then $s$ is a minimal simple element for $X$ if and only if it is a minimal simple element for $X^{-1}$.

Proof. By definition, $\ell(X)>0$. If $\ell(X)=1$ then $\operatorname{USS}\left(X^{-1}\right)=\left\{y^{-1} \mid y \in\right.$ $\operatorname{USS}(X)\}$ follows from Lemma 3.7 and Remark 1.8. In the case $\ell(X)>1$ the set $\operatorname{USS}(X)$ is made of rigid elements by Proposition 3.3 and $\operatorname{USS}\left(X^{-1}\right)=\left\{y^{-1} \mid y \in\right.$ $\operatorname{USS}(X)\}$ by Corollary 3.5 .

Hence, if $s$ is a simple element such that $s^{-1} X s \in \operatorname{USS}(X)$, then $s^{-1} X s$ either has canonical length 1 , or is rigid. In either case, $s^{-1} X^{-1} s$ satisfies the same property, so $s^{-1} X^{-1} s \in \operatorname{USS}\left(X^{-1}\right)$. Hence the set of simple elements conjugating $X$ to an element in USS $(X)$ coincides with the set of simple elements conjugating $X^{-1}$ to an element in USS $\left(X^{-1}\right)$. Since the minimal simple elements for $X$ and for $X^{-1}$ are the $\preccurlyeq$-minimal elements in these sets, it follows that the sets of minimal simple elements for $X$ and for $X^{-1}$ coincide.

Corollary 3.9. If $X$ is rigid and $s$ is a minimal simple element for $X$, then $s$ is a black [grey] arrow in $\Gamma_{X}$ if and only if it is a grey [black] arrow in $\Gamma_{X^{-1}}$.

Proof. By the above result, $s$ is an arrow in $\Gamma_{X}$ starting at $X$ if and only if it is also an arrow in $\Gamma_{X^{-1}}$ starting at $X^{-1}$. The statement about the color of the arrow is then given by definition.

Corollary 3.10. If $X$ is rigid and $\ell(X)>1$, then $\Gamma_{X}$ and $\Gamma_{X^{-1}}$ are isomorphic graphs, with the same labels but interchanged colors.

Proof. By Corollary 3.5 we have USS $\left(X^{-1}\right)=\left\{y^{-1} \mid y \in \operatorname{USS}(X)\right\}$ and by Proposition 3.3, every element in USS $(X)$ is rigid, so Corollary 3.9 can be applied to every vertex in $\Gamma_{X}$. Hence, an isomorphism between $\Gamma_{X}$ and $\Gamma_{X^{-1}}$ can be defined by sending every vertex $Y$ to $Y^{-1}$, and every arrow $s$ to itself. It is an isomorphism since every arrow $s$ in $\Gamma_{X}$ starting at $Y$ and ending at $Z$ (thus $s^{-1} Y s=Z$ ) is sent to an arrow $s$ in $\Gamma_{X^{-1}}$ starting at $Y^{-1}$, whose final vertex is precisely $s^{-1} Y^{-1} s=Z^{-1}$. 
Example 3.11. We return to the example that we gave earlier, in Figure 1. We included all edge labels, for easy comparison between the two graphs. Recall that $\bar{A}=A^{-1}$. The reader will see that the two black cycling orbits of length 3 in $\Gamma_{A}$ correspond to two grey orbits in $\Gamma_{\bar{A}}$, whereas the black cycling orbit of length 6 in $\Gamma_{\bar{A}}$ corresponds to a grey orbit of length 6 in $\Gamma_{A}$. The correspondence between vertex labels in $\Gamma_{A}$ and $\Gamma_{\bar{A}}$ is as follows: $A_{1,1}^{-1}=\bar{A}_{1,1}, A_{1,2}^{-1}=\bar{A}_{1,3}, A_{1,3}^{-1}=\bar{A}_{1,5}, A_{2,1}^{-1}=\bar{A}_{1,4}, A_{2,2}^{-1}=\bar{A}_{1,6}$, $A_{2,3}^{-1}=\bar{A}_{1,2}$.

In the case of rigid elements, there is a characterization of black and grey arrows which does not hold in the general case.

Proposition 3.12. If $X$ is rigid and $s$ is a minimal simple element for $X$, then the following conditions are equivalent.

(1) $s$ is a black arrow in $\Gamma_{X}$ (that is, $s \preccurlyeq \iota(X)$ ).

(2) $\varphi\left(X^{-1}\right) s$ is simple.

(3) $\varphi(X) s$ is left weighted.

(4) $s \wedge \iota\left(X^{-1}\right)=1$.

The equivalence of conditions (1) and (2) and the equivalence of conditions (3) and (4) hold for arbitrary $X \in \operatorname{USS}(X)$.

Proof. For arbitrary $X \in \operatorname{USS}(X)$, the product $\varphi(X) s$ is left weighted if and only if $s \wedge \partial(\varphi(X))=1$. By Lemma 2.1, we know that $\partial(\varphi(X))=\iota\left(X^{-1}\right)$, hence conditions (3) and (4) are equivalent. Moreover, $\iota(X)=\partial\left(\varphi\left(X^{-1}\right)\right)$, so $s$ is black if and only if $s \preccurlyeq \partial\left(\varphi\left(X^{-1}\right)\right)$ or, in other words, if and only if $\varphi\left(X^{-1}\right) s$ is simple. Hence, conditions (1) and (2) are equivalent.

If $X$ is rigid, we know by Theorem 2.5 that $\varphi(X) s$ is either simple or left weighted. By Lemma 3.2 we know that $s$ is black if and only if it is not grey, which happens, since $\iota\left(X^{-1}\right)=\partial(\varphi(X))$, if and only if $\varphi(X) s$ is not simple. Therefore, conditions (1) and (3) are equivalent.

Proposition 3.13. If $X$ is rigid and $s$ is a minimal simple element for $X$, then the following conditions are equivalent.

(1) $s$ is a grey arrow in $\Gamma_{X}$ (that is, $\left.s \preccurlyeq \iota\left(X^{-1}\right)\right)$.

(2) $\varphi(X) s$ is simple.

(3) $\varphi\left(X^{-1}\right) s$ is left-weighted.

(4) $s \wedge \iota(X)=1$.

The equivalence of conditions (1) and (2) and the equivalence of conditions (3) and (4) hold for arbitrary $X \in \operatorname{USS}(X)$. 
Proof. The equivalence of conditions (1) and (2) and the equivalence of conditions (3) and (4) can be shown as in the proof of Proposition 3.12 with $X$ replaced by $X^{-1}$. The rest is a consequence of Proposition 3.12, together with Corollary 3.9.

3.2. Paths in the USS graph of a rigid element. In the general case, we saw how two elements in USS $(X)$ are always joined by an oriented black path followed by an oriented grey path, and vice versa. In the rigid case, we will see that we can say much more. First, we can characterize black or grey oriented paths according to their associated conjugating element.

Lemma 3.14. Let $X \in G$ be rigid, and $\ell(X)>1$. Let $\left(s_{1}, \ldots, s_{k}\right)$ be an oriented path in $\Gamma_{X}$ starting at a vertex $Y$, and let $\alpha=s_{1} \ldots s_{k}$. The following properties are equivalent:

(1) The path $\left(s_{1}, \ldots, s_{k}\right)$ is black.

(2) $\alpha \preccurlyeq\left(Y^{m}\right)^{\circ}$ for some positive integer $m$.

(3) $\alpha \wedge \iota\left(Y^{-1}\right)=1$.

Proof. We will show the result by induction on $k$. The case $k=0$ is trivial. If $k=1$ then $\alpha=s_{1}$ is simple, hence the result follows from the definition of a black arrow, since in this case $s_{1} \preccurlyeq\left(Y^{m}\right)^{\circ}$ if and only if $s_{1} \preccurlyeq \iota\left(\left(Y^{m}\right)^{\circ}\right)=\iota\left(Y^{m}\right)$. Note that $Y$ is rigid by Proposition 3.3. Therefore $\iota\left(Y^{m}\right)=\iota(Y)$, hence $s_{1} \preccurlyeq\left(Y^{m}\right)^{\circ}$ if and only if $s_{1} \preccurlyeq \iota(Y)$, that is, if and only if $s_{1}$ is a black arrow. Hence Properties (1) and (2) are equivalent if $k=1$. In the case $\alpha=s_{1}$, Property (3) just means that $s_{1}$ is not a grey arrow, which by Lemma 3.2 and the rigidity of $Y$, is equivalent to Property (1).

Suppose that $k>1$ and that the result is true for $k-1$. Let $\Delta^{p} y_{1} \ldots y_{r}$ be the left normal form of $Y$ and define $Z=s_{1}^{-1} Y s_{1}$. Notice that $\left(s_{2}, \ldots, s_{k}\right)$ is an oriented path starting at $Z$. Notice also that, since $Z \in \operatorname{USS}(X)$, one has $Z^{\circ}=$ $s_{1}^{-1} Y^{\circ} \tau^{-p}\left(s_{1}\right)$. Moreover, we know by Proposition 3.3 that $Y$ and $Z$ are rigid, so $Y^{m}$ and $Z^{m}$ are also rigid, hence $Y^{m}, Z^{m} \in \operatorname{USS}\left(X^{m}\right)$ for every $m>0$. Therefore $\left(Z^{m}\right)^{\circ}=\left(s_{1}^{-1} Y^{m} s_{1}\right)^{\circ}=s_{1}^{-1}\left(Y^{m}\right)^{\circ} \tau^{-p m}\left(s_{1}\right)$ for every $m>0$.

By induction hypothesis we have that $\left(s_{2}, \ldots, s_{k}\right)$ is a black path if and only if $s_{2} \ldots s_{k} \preccurlyeq\left(Z^{m}\right)^{\circ}$ for some positive $m$. Therefore, if $\left(s_{1} \ldots, s_{k}\right)$ is a black path, then $\alpha=s_{1} s_{2} \ldots s_{k} \preccurlyeq s_{1}\left(Z^{m}\right)^{\circ}=s_{1} s_{1}^{-1}\left(Y^{m}\right)^{\circ} \tau^{-p m}\left(s_{1}\right)=\left(Y^{m}\right)^{\circ} \tau^{-p m}\left(s_{1}\right)$. But $s_{1}$ is a black arrow, hence $s_{1} \preccurlyeq \iota(Y)=\tau^{-p}\left(y_{1}\right)$, so $\left(Y^{m}\right)^{\circ} \tau^{-p m}\left(s_{1}\right) \preccurlyeq$ $\left(Y^{m}\right)^{\circ} \tau^{-p(m+1)}\left(y_{1}\right) \preccurlyeq\left(Y^{m+1}\right)^{\circ}$, where the last claim is true due to the rigidity of $Y$. Therefore, if $\left(s_{1}, \ldots, s_{k}\right)$ is a black path, then $\alpha \preccurlyeq\left(Y^{m+1}\right)^{\circ}$ for some positive $m$, so Property (1) implies Property (2).

Conversely, suppose that $\alpha \preccurlyeq\left(Y^{m}\right)^{\circ}$ for some positive integer $m$. Then $s_{1} \preccurlyeq$ $\alpha \preccurlyeq\left(Y^{m}\right)^{\circ}$, and since $s_{1}$ is simple $s_{1} \preccurlyeq \iota\left(\left(Y^{m}\right)^{\circ}\right)=\iota\left(Y^{m}\right)=\iota(Y)$, whence $s_{1}$ is a black arrow. Moreover, $\alpha=s_{1} \ldots s_{k} \preccurlyeq\left(Y^{m}\right)^{\circ} \preccurlyeq\left(Y^{m}\right)^{\circ} \tau^{-m p}\left(s_{1}\right)$, so $s_{2} \ldots s_{k} \preccurlyeq s_{1}^{-1}\left(Y^{m}\right)^{\circ} \tau^{-m p}\left(s_{1}\right)=\left(Z^{m}\right)^{\circ}$. By induction hypothesis this means that 
$\left(s_{2}, \ldots, s_{k}\right)$ is a black path, hence $\left(s_{1}, \ldots, s_{k}\right)$ is a black path, that is, Property (2) implies Property (1). The first two properties are thus equivalent.

Now suppose again that $\alpha \preccurlyeq\left(Y^{m}\right)^{\circ}$ for some positive $m$. In particular, one has $\inf (\alpha)=0$, so it follows that $\iota(\alpha) \preccurlyeq \iota\left(\left(Y^{m}\right)^{\circ}\right)=\iota\left(Y^{m}\right)=\iota(Y)$, where the last equality follows from the rigidity of $Y$. Since $Y$ is rigid, $\iota(Y) \wedge \iota\left(Y^{-1}\right)=1$, hence $\iota(\alpha) \wedge \iota\left(Y^{-1}\right)=1$ and therefore $\alpha \wedge \iota\left(Y^{-1}\right)=1$. This shows that Property (2) implies Property (3).

Finally, suppose that $\alpha \wedge \iota\left(Y^{-1}\right)=1$. This implies that $\inf (\alpha)=0$, so the left normal form of $\alpha$ has the form $\alpha_{1} \ldots \alpha_{t}$. Since $\alpha \wedge \iota\left(Y^{-1}\right)=\alpha \wedge \partial\left(y_{r}\right)=1$, the left normal form of $Y \alpha$ is precisely $\Delta^{p} y_{1} \ldots y_{r} \alpha_{1} \ldots \alpha_{t}$. Moreover, since $Y$ is rigid, it follows that for every $m>0$ the left normal form of $Y^{m} \alpha$ is

$$
\begin{gathered}
Y^{m} \alpha=\Delta^{p m} \tau^{p(m-1)}\left(y_{1}\right) \ldots \tau^{p(m-1)}\left(y_{r}\right) \tau^{p(m-2)}\left(y_{1}\right) \ldots \tau^{p(m-2)}\left(y_{r}\right) \ldots \\
\ldots y_{1} \ldots y_{r} \alpha_{1} \ldots \alpha_{t} .
\end{gathered}
$$

That is, there is no contribution of $\alpha$ to the first $m r$ non- $\Delta$ factors of the left normal form of $Y^{m} \alpha$. However, $\alpha^{-1} Y^{m} \alpha=Z^{m} \in \operatorname{USS}\left(X^{m}\right)$, whence $\alpha \preccurlyeq\left(Y^{m} \alpha\right)^{\circ}$ for every $m>0$. This means that, for $m$ big enough (such that $t \leq m r$ ) one has $\alpha \preccurlyeq\left(Y^{m}\right)^{\circ}$. Hence Property (3) implies Property (2), thus the last two properties are also equivalent.

Here is the analogous result for grey paths.

Lemma 3.15. Let $X \in G$ be rigid, and $\ell(X)>1$. Let $\left(s_{1}, \ldots, s_{k}\right)$ be an oriented path in $\Gamma_{X}$ starting at a vertex $Y$, and let $\alpha=s_{1} \ldots s_{k}$. Then the following properties are equivalent:

(1) The path $\left(s_{1}, \ldots, s_{k}\right)$ is grey.

(2) $\alpha \preccurlyeq\left(Y^{-m}\right)^{\circ}$ for some positive integer $m$.

(3) $\alpha \wedge \iota(Y)=1$.

Proof. By Corollary 3.10 this is analogous to the above result, considering the graph $\Gamma_{X^{-1}}$ instead of $\Gamma_{X}$.

In the particular case where $\alpha$ above is simple, the characterization of black or grey paths can be made more precise.

Corollary 3.16. Let $X \in G$ be rigid and $\ell(X)>1$. Let $\left(s_{1}, \ldots, s_{k}\right)$ be an oriented path in $\Gamma_{X}$ starting at a vertex $Y$ and let $s=s_{1} \ldots s_{k}$. If $s$ is simple, the following properties are equivalent:

(1) The path $\left(s_{1}, \ldots, s_{k}\right)$ is black.

(2) $s \preccurlyeq \iota(Y)$. 
(3) $s \wedge \iota\left(Y^{-1}\right)=1$.

(4) $\varphi\left(Y^{-1}\right) s$ is simple.

Proof. The equivalence of Properties (1)-(3) is a consequence of Lemma 3.14, together with the fact that, if $s$ is simple and $Y$ is rigid, $s \preccurlyeq\left(Y^{m}\right)^{\circ}$ if and only if $s \preccurlyeq \iota\left(Y^{m}\right)^{\circ}=\iota(Y)$. The equivalence of Properties (2) and (4) follows from $\iota(Y)=\partial\left(\varphi\left(Y^{-1}\right)\right)$.

Corollary 3.17. Let $X \in G$ be rigid, and $\ell(X)>1$. Let $\left(s_{1}, \ldots, s_{k}\right)$ be an oriented path in $\Gamma_{X}$ starting at a vertex $Y$, and let $s=s_{1} \ldots s_{k}$. If $s$ is simple, the following properties are equivalent:

(1) The path $\left(s_{1}, \ldots, s_{k}\right)$ is grey.

(2) $s \preccurlyeq \iota\left(Y^{-1}\right)$.

(3) $s \wedge \iota(Y)=1$.

(4) $\varphi(Y) s$ is simple.

Proof. This result is the same as the above one, but applied to $\Gamma_{X^{-1}}$ instead of $\Gamma_{X}$, thanks to Corollary 3.10 .

Remark 3.18. Using the same kind of proofs as above, one can show that Corollary 3.16 and Corollary 3.17 are also true if $Y$ is rigid and $\ell(Y)=1$. But this cannot be generalized to paths whose associated element is not simple, so we will keep considering rigid elements of canonical length greater than 1 .

Now recall that we showed, in the general case, that every oriented path could be transformed into the concatenation of a black path and a grey path, whose associated element was the same as the original one. We will now see that, if $X$ is rigid and $\ell(X)>1$, the elements associated to those oriented black and grey paths are, in fact, determined by the associated element of the whole path.

Lemma 3.19. Let $X$ be a rigid element with canonical length $\ell(X)=r>1$ and let $\left(b_{1}, \ldots, b_{s}, g_{1}, \ldots, g_{t}\right)$ be an oriented path in $\Gamma_{X}$ starting at $Y$, such that $\left(b_{1}, \ldots, b_{s}\right)$ is a black path and $\left(g_{1}, \ldots, g_{t}\right)$ is a grey one. Let $b=b_{1} \ldots b_{s}, g=g_{1} \ldots g_{t}$, and $\alpha=b g$. Let $m$ be an integer big enough such that $\ell(b) \leq m r$. Then, $b=\left(Y^{m}\right)^{\circ} \wedge \alpha$. In particular, $b$ and $g$ are uniquely determined by $\alpha$.

Proof. Since $\left(b_{1}, \ldots, b_{s}\right)$ is a black path, Lemma 3.14 tells us that $b \preccurlyeq\left(Y^{m}\right)^{\circ}$ for some $m$ big enough. Actually, by the rigidity of $Y$, it suffices to take $m$ such that $\ell(b) \leq \ell\left(\left(Y^{m}\right)^{\circ}\right)=m r$. Since we also have $b \preccurlyeq b g=\alpha$, it follows that $b \preccurlyeq\left(Y^{m}\right)^{\circ} \wedge \alpha$. 
Now let $Z=b^{-1} Y b$ and $p=\inf (Y)$. Since $Z$ is also rigid, both $Y^{m}$ and $Z^{m}$ belong to $\operatorname{USS}\left(X^{m}\right)$. Hence $\left(Z^{m}\right)^{\circ}=b^{-1}\left(Y^{m}\right)^{\circ} \tau^{-p m}(b)$. Moreover, $\iota\left(\left(Z^{m}\right)^{\circ}\right)=$ $\iota(Z)$. However, $\left(g_{1}, \ldots, g_{t}\right)$ is a grey path starting at $Z$, hence by Lemma 3.15 $g \wedge \iota(Z)=g \wedge \iota\left(\left(Z^{m}\right)^{\circ}\right)=1$. Since $\inf \left(\left(Z^{m}\right)^{\circ}\right)=0$, this implies that $g \wedge\left(Z^{m}\right)^{\circ}=1$, that is, $g \wedge\left(b^{-1}\left(Y^{m}\right)^{\circ} \tau^{-p m}(b)\right)=1$. As $b \preccurlyeq\left(Y^{m}\right)^{\circ}$, we in particular have $g \wedge$ $b^{-1}\left(Y^{m}\right)^{\circ}=1$. Multiplying on the left by $b$, one gets $\alpha \wedge\left(Y^{m}\right)^{\circ}=b$, as we wanted to show.

The analogous result holds for the concatenation of a grey path followed by a black path.

Lemma 3.20. Let $X$ be a rigid element with canonical length $\ell(X)=r>1$ and let $\left(g_{1}, \ldots, g_{t}, b_{1}, \ldots, b_{s}\right)$ be an oriented path in $\Gamma_{X}$ starting at $Y$, such that $\left(g_{1}, \ldots, g_{t}\right)$ is a grey path and $\left(b_{1}, \ldots, b_{s}\right)$ is a black one. Let $g=g_{1} \ldots g_{t}, \quad b=b_{1} \ldots b_{s}$, and $\alpha=g b$. Let $m$ be an integer big enough such that $\ell(g) \leq m r$. Then, $g=\left(Y^{-m}\right)^{\circ} \wedge \alpha$. In particular, $g$ and $b$ are uniquely determined by $\alpha$.

Proof. Corollary 3.10 implies that this result is equivalent to the previous one.

In the case that $\alpha$ above is simple, one can simplify the characterization of the elements $b$ and $g$. We will state the two analogous results since we will use them later.

Corollary 3.21. Let $X$ be a rigid element with canonical length $\ell(X)>1$ and let $\left(b_{1}, \ldots, b_{s}, g_{1}, \ldots, g_{t}\right)$ be an oriented path in $\Gamma_{X}$ starting at $Y$, such that $\left(b_{1}, \ldots, b_{s}\right)$ is a black path and $\left(g_{1}, \ldots, g_{t}\right)$ is a grey one. Let $b=b_{1} \ldots b_{s}, g=g_{1} \ldots g_{t}$ and $s=b g$. If $s$ is simple, then $b=\iota(Y) \wedge s$. In particular, $b$ and $g$ are uniquely determined by $s$.

Proof. This follows from Lemma 3.19, since by the simplicity of $s$ and the rigidity of $Y$, one has $b=\left(Y^{m}\right)^{\circ} \wedge s=\Delta \wedge\left(Y^{m}\right)^{\circ} \wedge s=\iota\left(\left(Y^{m}\right)^{\circ}\right) \wedge s=\iota\left(Y^{m}\right) \wedge s=\iota(Y) \wedge s$.

Corollary 3.22. Let $X$ be a rigid element with canonical length $\ell(X)>1$ and let $\left(g_{1}, \ldots, g_{t}, b_{1}, \ldots, b_{s}\right)$ be an oriented path in $\Gamma_{X}$ starting at $Y$, such that $\left(g_{1}, \ldots, g_{t}\right)$ is a grey path and $\left(b_{1}, \ldots, b_{s}\right)$ is a black one. Let $g=g_{1} \ldots g_{t}, \quad b=b_{1} \ldots b_{s}$ and $s=g b$. If $s$ is simple, then $g=\partial(\varphi(Y)) \wedge s$, that is, the left normal form of $\varphi(Y) s$ is $(\varphi(Y) g) b$. In particular, $g$ and $b$ are uniquely determined by $s$.

Proof. This follows by applying Corollary 3.21 to $\Gamma_{X^{-1}}$.

Remark. In the above results, the elements $b$ and $g$ are determined by $\alpha$ (or $s$ ). But this does not mean that the paths $\left(b_{1}, \ldots, b_{s}\right)$ and $\left(g_{1}, \ldots, g_{t}\right)$ are determined 
by $\alpha$, since there could exist distinct paths $\left(b_{1}, \ldots, b_{s}\right)$ and $\left(b_{1}^{\prime}, \ldots, b_{u}^{\prime}\right)$ such that $b=b_{1} \ldots b_{s}=b_{1}^{\prime} \ldots b_{u}^{\prime}$. The same could happen for $g$.

The situation is more interesting if each of the above paths consists only of one arrow. Consider then the following situation. Let $X=\Delta^{p} x_{1} \ldots x_{r}, \quad Y=\Delta^{p} y_{1} \ldots y_{r}$ and $Z=\Delta^{p} z_{1} \ldots z_{r}$ be three vertices of $\Gamma_{X}$, where $X$ is rigid and $\ell(X)>1$. Suppose that $X$ and $Y$ are joined by a black arrow $b$, while $Y$ and $Z$ are joined by arey arrow $g$. That is,

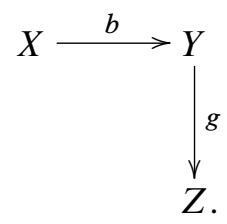

Proposition 3.23. In the above situation, the product $b g$ is simple, and there exist a unique grey arrow $g^{\prime}$ and a unique black arrow $b^{\prime}$ such that $b g=g^{\prime} b^{\prime}$ :

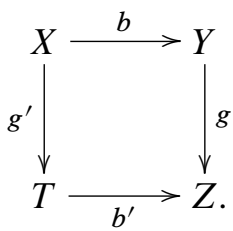

Specifically, $g^{\prime}=\partial(\varphi(X)) \wedge b g$ and $b^{\prime}=\left(g^{\prime}\right)^{-1} b g$.

Proof. Since $b \preccurlyeq \iota(X)$ ( $b$ is a black arrow), one can decompose $\iota(X)=b c$ so that $Y=\Delta^{p} \tau^{p}(c) x_{2} \ldots x_{r} b$, where $x_{2} \ldots x_{r} b$ is in left normal form as written, by the rigidity of $X$. Hence, when we compute the left normal form of $Y$, we see that $y_{r}=\omega b$ for some $\omega$ such that $x_{r} \geqslant \omega$. However, $g$ is a grey arrow for $Y$, hence $y_{r} g=\omega b g$ is simple, thus $b g$ is also simple.

Consider $b^{\prime}$ and $g^{\prime}$ as defined above. We know that, since $(b, g)$ is an oriented path going from $X$ to $Z$, there exists another path $\left(g_{1}, \ldots, g_{t}, b_{1}, \ldots, b_{s}\right)$ also going from $X$ to $Z$, which is the concatenation of a grey path and a black path, such that $b g=$ $g_{1} \ldots g_{t} b_{1} \ldots b_{s}$. Moreover, by Corollary 3.22, $g^{\prime}=g_{1} \ldots g_{t}$ and $b^{\prime}=b_{1} \ldots b_{s}$. We just need to show that $t=s=1$, that is, $g^{\prime}=g_{1}$ and $b^{\prime}=b_{1}$.

Firstly, we cannot have $t=0$ or $s=0$, since otherwise we would have an oriented black path, or an oriented grey path, whose associated element is $b g$. But this is not possible, since in that case $(b, g)$ would be either a black path or a grey path, by Lemma 3.14 or Lemma 3.15, and we know this is not true.

Suppose now that $t>1$. Then $\left(g_{t}, b_{1}, \ldots, b_{s}\right)$ is a path which has black and grey arrows, that can be transformed into a path $\left(b_{1}^{\prime}, \ldots, b_{u}^{\prime}, g_{1}^{\prime}, \ldots, g_{v}^{\prime}\right)$ where every $b_{i}^{\prime}$ is black, every $g_{i}^{\prime}$ is grey, and $v>0$ by the above reasoning. There- 
fore $\left(g_{1}, \ldots, g_{t-1}, b_{1}^{\prime}, \ldots, b_{u}^{\prime}, g_{1}^{\prime}, \ldots, g_{v}^{\prime}\right)$ is a path going from $X$ to $Z$. Now $\left(g_{1}, \ldots, g_{t-1}, b_{1}^{\prime}, \ldots, b_{u}^{\prime}\right)$ can also be replaced by a path $\left(b_{1}^{\prime \prime}, \ldots, b_{\mu}^{\prime \prime}, g_{1}^{\prime \prime}, \ldots, g_{v}^{\prime \prime}\right)$, where every $b_{i}^{\prime \prime}$ is black, every $g_{i}^{\prime \prime}$ is grey, and $v>0$. Hence, if $t>1$ we could obtain a path $\left(b_{1}^{\prime \prime}, \ldots b_{\mu}^{\prime \prime}, g_{1}^{\prime \prime}, \ldots, g_{v}^{\prime \prime}, g_{1}^{\prime}, \ldots, g_{v}^{\prime}\right)$, whose associated element is $b g$, and $v+v>1$. But by Lemma 3.19, $g=g_{1}^{\prime \prime} \ldots g_{v}^{\prime \prime} g_{1}^{\prime} \ldots g_{v}^{\prime}$, that is, the grey arrow $g$ could be decomposed into a product of more than one grey arrow. This contradicts the fact that $g$ is a minimal simple element, since in that case $g_{1}^{\prime \prime}$ would be a proper prefix of $g$. Therefore $t=1$.

We can use the same reasoning to show that $s=1$, so $\left(g^{\prime}, b^{\prime}\right)$ is an oriented path, where $g^{\prime}$ is a grey arrow and $b^{\prime}$ is a black arrow. The uniqueness of $g^{\prime}$ and $b^{\prime}$ follows from Lemma 3.20.

We have then shown how to transform any black-grey path $(b, g)$ into a grey-black path $\left(g^{\prime}, b^{\prime}\right)$ such that $b g=g^{\prime} b^{\prime}$. Moreover, by Corollary 3.21 and Corollary 3.22, these two decompositions $b g=g^{\prime} b^{\prime}$ are the only two ways to decompose $b g$ into a product of minimal simple elements.

By symmetry, if we start with a grey-black path $(g, b)$, we can transform it into a black-grey path $\left(b^{\prime}, g^{\prime}\right)$, where $g b=b^{\prime} g^{\prime}$.

Proposition 3.24. Let $X \in G$ be rigid and $\ell(X)>1$. Let $(g, b)$ be a path in $\Gamma_{X}$ starting at $X$, where $g$ is a grey arrow and $b$ is a black one. If we define $b^{\prime}=g b \wedge \iota(X)$ and $g^{\prime}=\left(b^{\prime}\right)^{-1} g b$, then $\left(b^{\prime}, g^{\prime}\right)$ is a path in $\Gamma_{X}$ such that $b^{\prime}$ is black, $g^{\prime}$ is grey, and $g b=b^{\prime} g^{\prime}$. Moreover, $b^{\prime}$ and $g^{\prime}$ are the unique such arrows.

Proof. The result follows by applying Proposition 3.23 to the graph $\Gamma_{X^{-1}}$ and using $\partial\left(\varphi\left(X^{-1}\right)\right)=\iota(X)$.

We have thus shown that, if $X$ and $Y$ are two rigid elements connected by a black arrow, then every grey arrow for $Y$ is related to a grey arrow for $X$. However, we do not yet know whether every grey arrow of $X$ is related to one of $Y$. Similarly, if $X$ and $Y$ are two rigid elements connected by a grey arrow, the question arises of whether every black arrow of $X$ is related to one of $Y$.

Using the language of Proposition 3.23, we need to show that given a black arrow $b$ and a grey arrow $g^{\prime}$ for $X$, a grey arrow $g$ for $X^{b}$ and a black arrow $b^{\prime}$ for $X^{g^{\prime}}$ exist such that $\left(X^{b}\right)^{g}=\left(X^{g^{\prime}}\right)^{b^{\prime}}$. In the next subsection, we will see that this is true.

As a consequence, we will show that every two elements in the same black component have the same number of grey arrows and that every two elements in the same grey component have the same number of black arrows.

3.3. Partial transport. Let $X=\Delta^{p} x_{1} \ldots x_{r} \in G$ be in left normal form, such that $X \in \operatorname{SSS}(X)$. We start this subsection by recalling the definition of the transport of a simple element, given in [18]. Let $s$ be a simple element such that $s^{-1} X s=Y \in$ 
$\operatorname{SSS}(X)$. We can write this by $X \stackrel{s}{\rightarrow} Y$. We know from $[14, \S 4]$ that $\mathbf{c}(X)$ and $\mathbf{c}(Y)$ also belong to $\operatorname{SSS}(X)$. Notice that $X \stackrel{\iota(X)}{\longrightarrow} \mathbf{c}(X)$ and $Y \stackrel{\iota(Y)}{\longrightarrow} \mathbf{c}(Y)$. In [18, Lemma 2.3] it is shown that, in this situation, $\mathbf{c}(X)$ and $\mathbf{c}(Y)$ are also conjugate by a simple element, $s^{(1)}$, where $s^{(1)}$ is defined as the element making the following diagram commutative in the sense explained below:

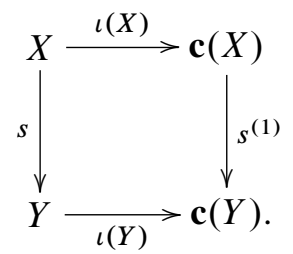

More precisely, $s^{(1)}=\iota(X)^{-1} s \iota(Y)$. The nontrivial fact shown in [18, Corollary 2.2] is that $s^{(1)}$ is simple. The simple element $s^{(1)}$ is the transport of $s$.

Remark. When we deal with a diagram such as the previous one, in which the arrows represent conjugating elements, then by saying that the diagram is commutative we mean the following: for every two paths in the diagram whose starting and ending vertices coincide, the products of the arrows forming every path (with the corresponding signs) are the same. For instance, in the above diagram this is equivalent to $\iota(X) s^{(1)}=s \iota(Y)$, that is, $s^{(1)}=\iota(X)^{-1} s \iota(Y)$.

We can then define the transport of $s^{(1)}$, denoted $s^{(2)}$, and so on. In general, the transport of $s^{(i-1)}$ is denoted $s^{(i)}$ and the above definition tells us that

$$
s^{(i)}=\iota\left(\mathbf{c}^{i-1}(X)\right)^{-1} \ldots \iota(\mathbf{c}(X))^{-1} \iota(X)^{-1} s \iota(Y) \iota(\mathbf{c}(Y)) \ldots \iota\left(\mathbf{c}^{i-1}(Y)\right) .
$$

We can easily see that, if $X \in \operatorname{USS}(X)$ is rigid and $\ell(X)>1$, the situation is much simpler. In this case, if the left normal form of $X$ is $\Delta^{p} x_{1} \ldots x_{r}$, that is $\iota(X)=\tau^{-p}\left(x_{1}\right)$, rigidity of $\mathbf{c}^{k}(X)$ for all $k \geq 0$ implies $\iota(\mathbf{c}(X))=\tau^{-p}\left(x_{2}\right)$, $\iota\left(\mathbf{c}^{2}(X)\right)=\tau^{-p}\left(x_{3}\right)$ and so on. We also know that $Y=\Delta^{p} y_{1} \ldots y_{r}$ must be rigid, so the same formulae hold for $Y$. Hence, for $i=1, \ldots, r$ we have

$$
s^{(i)}=\tau^{-p}\left(x_{i}^{-1} \ldots x_{1}^{-1}\right) s \tau^{-p}\left(y_{1} \ldots y_{i}\right) .
$$

Eventually, one has

$$
\begin{aligned}
s^{(r)} & =\tau^{-p}\left(x_{r}^{-1} \ldots x_{1}^{-1}\right) s \tau^{-p}\left(y_{1} \ldots y_{r}\right)=\Delta^{p} X^{-1} s Y \Delta^{-p} \\
& =\Delta^{p} X^{-1} s\left(s^{-1} X s\right) \Delta^{-p}=\Delta^{p} s \Delta^{-p}=\tau^{-p}(s) .
\end{aligned}
$$

Hence, if $m$ is an integer such that $p m$ is a multiple of $e$ (where $\Delta^{e}$ is central), one has $s^{(r m)}=\tau^{p m}(s)=s$. For instance, in braid groups with the Garside structure given by the Artin generators, one has $e=2$, so we always have $s^{(2 r)}=s$. If $p$ is even, $s^{(r)}=s$ holds. 
There is another way of looking at the transports of $s$ in the rigid case. By definition, we have $s^{(1)}=\tau^{-p}\left(x_{1}\right)^{-1} s \tau^{-p}\left(y_{1}\right)$, whence $y_{1}=\tau^{p}\left(s^{-1}\right) x_{1} \tau^{p}\left(s^{(1)}\right)$. But since $X$ and $Y$ are rigid, we have $\iota(\mathbf{c}(X))=\tau^{-p}\left(x_{2}\right)$ and $\iota(\mathbf{c}(Y))=\tau^{-p}\left(y_{2}\right)$, whence, $y_{2}=\tau^{p}\left(s^{(1)}\right)^{-1} x_{2} \tau^{p}\left(s^{(2)}\right)$. In general, $y_{i}=\tau^{p}\left(s^{(i-1)}\right)^{-1} x_{i} \tau^{p}\left(s^{(i)}\right)$ for $i=1, \ldots, r$. Recalling $s^{(r)}=\tau^{-p}(s)$, the normal form of $Y$ is

$$
\begin{aligned}
Y & =\Delta^{p} y_{1} y_{2} \ldots y_{r} \\
& =\Delta^{p}\left(\tau^{p}(s)^{-1} x_{1} \tau^{p}\left(s^{(1)}\right)\right)\left(\tau^{p}\left(s^{(1)}\right)^{-1} x_{2} \tau^{p}\left(s^{(2)}\right)\right) \ldots\left(\tau^{p}\left(s^{(r-1)}\right)^{-1} x_{r} s\right) .
\end{aligned}
$$

Hence, in the rigid case, the first $r$ transports of $s$ are precisely the simple elements that tell us how to relate the left normal forms of $X$ and $Y$ (conjugated by $\Delta^{-p}$ ).

By [18, Corollary 2.7] the transport is a bijection from the minimal simple elements for $X$ to the minimal simple elements for $\mathbf{c}(X)$. Hence the map $\phi$ from the graph $\Gamma_{X}$ to itself defined by sending every vertex $Y$ to $\mathbf{c}(Y)$ and every arrow $s$ to $s^{(1)}$ is an automorphism of $\Gamma_{X}$. It is also shown in [18, Corollary 2.2] that $s \preccurlyeq t$ implies $s^{(1)} \preccurlyeq t^{(1)}$ and by definition $(\iota(Y))^{(1)}=\iota(\mathbf{c}(Y))$. Again by definition this means that $\phi$ sends black arrows to black arrows. It is elementary to check that the image of a grey arrow is a grey arrow. Hence $\phi$ is an automorphism of $\Gamma_{X}$ preserving the colors of arrows.

Example 3.25. Recall that the braids we called $A$ and $B$, with $\Gamma_{A}$ illustrated in the left sketch in Figure 1 and $\Gamma_{B}$ illustrated in Figure 2 are both rigid braids. Both will give us examples of the transport. We consider $A$ first. It has two cycling orbits, $A_{1}$ and $A_{2}$, with 3 elements each. Let $s$ be the grey arrow from $A_{1,1} \rightarrow A_{2,3}$. Then the transport of $s$ must be a grey arrow from $\mathbf{c}\left(A_{1,1}\right)=A_{1,2}$ to $\mathbf{c}\left(A_{2,3}\right)=A_{2,1}$, and indeed such an arrow exists and is labeled by the simple element $\sigma_{1} \sigma_{2}=s^{(1)}$.

Example $B$ is more complicated. Going back to Example 2.12 we study the two cycling orbits $B_{1}=\left\{B_{1,1}, B_{1,2}\right\}$ and $B_{2}=\left\{B_{2,1}, B_{2,2}\right\}$. Since $B_{1,1}$ and $B_{2,1}$ are rigid the data given in Example 2.12 tells us that

$$
\begin{aligned}
& B_{1,1}=\sigma_{2} \sigma_{1} \sigma_{4} \sigma_{3} \sigma_{2} \sigma_{1} \sigma_{5} \sigma_{4} \cdot \sigma_{2} \sigma_{4}, \\
& B_{1,2}=\sigma_{2} \sigma_{4} \cdot \sigma_{2} \sigma_{1} \sigma_{4} \sigma_{3} \sigma_{2} \sigma_{1} \sigma_{5} \sigma_{4}, \\
& B_{2,2}=\sigma_{2} \sigma_{4} \cdot \sigma_{2} \sigma_{1} \sigma_{3} \sigma_{4} \sigma_{3} \sigma_{2} \sigma_{5} \sigma_{4}, \\
& B_{2,1}=\sigma_{2} \sigma_{1} \sigma_{3} \sigma_{4} \sigma_{3} \sigma_{2} \sigma_{5} \sigma_{4} \cdot \sigma_{2} \sigma_{4},
\end{aligned}
$$

and that the conjugating element for cycling $\sigma_{2} \sigma_{1} \sigma_{4} \sigma_{3} \sigma_{2} \sigma_{1} \sigma_{5} \sigma_{4}$ which takes us from $B_{1,1} \rightarrow B_{1,2}$ is the product of the two black arrows $\sigma_{2} \sigma_{1}$ and $\sigma_{4} \sigma_{3} \sigma_{2} \sigma_{1} \sigma_{5} \sigma_{4}$ in Figure 2, both of which are minimal partial cyclings. Also, the conjugating element for cycling which takes us from $B_{2,2} \rightarrow B_{2,1}$ is $\sigma_{2} \sigma_{4}$, which is the same as the product of the two black arrows $\sigma_{4}$ and $\sigma_{2}$. We also see a grey arrow, call it $s$, from $B_{1,1} \rightarrow B_{2,2}$ and its label (which we did not compute explicitly in Example 2.12) is 
$s=\sigma_{1} \sigma_{2} \sigma_{3} \sigma_{2} \sigma_{1} \sigma_{4} \sigma_{3} \sigma_{2} \sigma_{5} \sigma_{4} \sigma_{3} \sigma_{2} \sigma_{1}$. The grey arrow from $B_{1,2}$ to $B_{2,1}$ has the label $t=\sigma_{3} \sigma_{2} \sigma_{1} \sigma_{4} \sigma_{5} \sigma_{4} \sigma_{3}$. One checks easily that this part of the diagram is commutative, so that $t=s^{(1)}$ is the transport of $s$.

Let us remember that each black arrow represents partial cycling. This immediately raises a question about the grey arrow that we see from $B_{3,1}$ to $B_{4,2}$. Is it, too, determined by a commutative diagram? That leads us to the title of this section: "Partial transport". It will take a while for us to define the concept precisely.

We will show that, in the rigid case, there is a notion of 'partial transport' of a minimal simple element, related to a partial cycling in the same way as the transport is related to the cycling. More precisely, we will see that a grey arrow $g$ starting at a vertex $X$ can be partially transported along a black arrow $b$, yielding a grey arrow $g[b]$ starting at $X^{b}$. Similarly, we will define the partial transport of $b$ along $g$, which is a black arrow $b^{[g]}$ starting at $X^{g}$. We will see that some natural properties are satisfied, and this will give us more information about the structure of $\Gamma_{X}$. In particular, we will show that every two vertices in the same black component have the same number of grey arrows, and vice versa.

Let $X=\Delta^{p} x_{1} \ldots x_{r}$ and $Y=\Delta^{p} y_{1} \ldots y_{r}$ be two vertices of $\Gamma_{X}$, where we assume that $X$ is rigid and $r>1$. Let $b$ be a black arrow going from $X$ to $Y$, that is $X \stackrel{b}{\longrightarrow} Y$ and $b \preccurlyeq \iota(X)$. We saw that when we conjugate $X$ by $b$ we are performing a partial cycling of $X$. Now we want to transport the grey arrows starting at $X$ along this partial cycling.

We have $\iota(X)=b c$ for some simple element $c$. Then $c$ conjugates $Y$ to $\mathbf{c}(X)$, and these two elements belong to $\operatorname{USS}(X)$. Therefore we can decompose $c=b_{2} \ldots b_{s}$ as a product of minimal simple elements. Denote $b=b_{1}$. Notice that $\iota(X)=b_{1} \ldots b_{s}$, where $\left(b_{1}, \ldots, b_{s}\right)$ is an oriented black path in $\Gamma_{X}$ by Corollary 3.16.

On the other hand, consider a grey arrow $g$ starting at $X$, and let $T=X^{g}$, whose left normal form is $\Delta^{p} t_{1} \ldots t_{r}$. We know that its transport $g^{(1)}$ is defined in such a way that the following diagram is commutative:

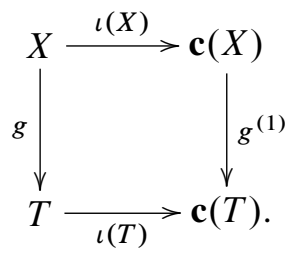

Moreover, since $g$ is a grey arrow starting at $X$, we have $\iota(X) \wedge g=1$ by Proposition 3.13. Hence, by [18, Corollary 2.2], $\iota(X)^{(1)} \wedge g^{(1)}=1$. As c $(X)=$ $X^{\iota(X)}$, the definition of the transport yields $\iota(X)^{(1)}=\iota(\mathbf{c}(X))$, whence $\iota(\mathbf{c}(X)) \wedge$ $g^{(1)}=1$. That is, $g^{(1)}$ is a grey arrow starting at $\mathbf{c}(X)$. 
Since we have decomposed $\iota(X)$ as a product of black arrows, the situation is the following:

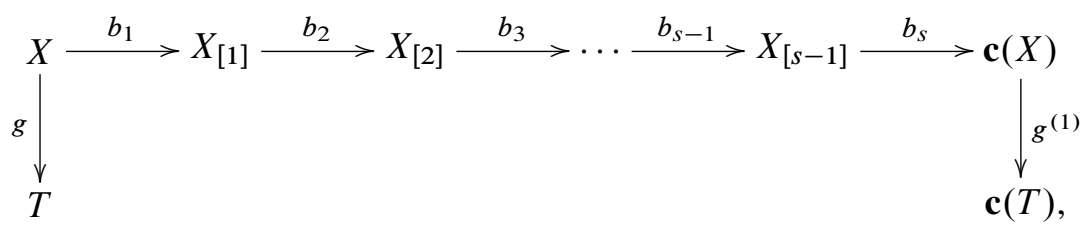

where $X_{[i]}$ is the conjugate of $X$ by $b_{1} \ldots b_{i}$. Notice that $X_{[1]}=Y$.

We saw in the previous subsection how to transform the black-grey path $\left(b_{s}, g^{(1)}\right)$ into a grey-black path $\left(g_{s-1}, b_{s}^{\prime}\right)$ such that the following diagram is commutative:

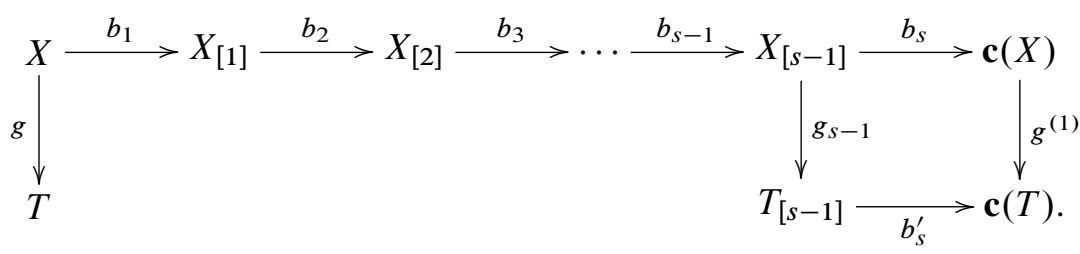

We can continue this process, defining for every black-grey path $\left(b_{i}, g_{i}\right)$ a new grey-black path $\left(g_{i-1}, b_{i}^{\prime}\right)$. At the end we will obtain the following commutative diagram:

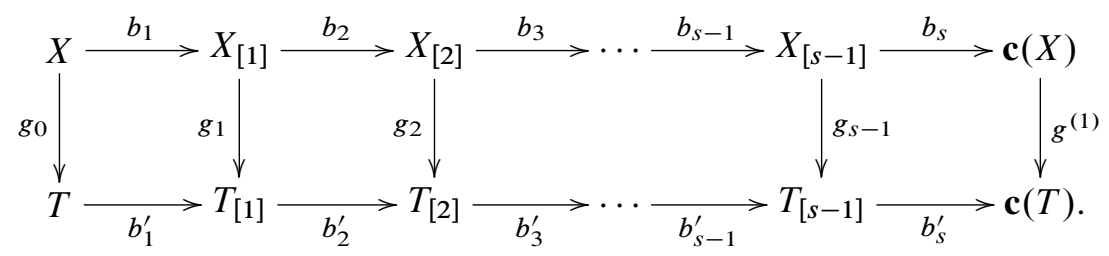

Lemma 3.26. In the above situation, $g_{0}=g$ and $b_{1}^{\prime} \ldots b_{s}^{\prime}=\iota(T)$.

Proof. Since $g^{(1)}$ is a grey arrow starting at $\mathbf{c}(X)$, we know by Proposition 3.13 that $\varphi(\mathbf{c}(X)) g^{(1)}$ is a simple element. Since $X$ is rigid, $\varphi(\mathbf{c}(X))=\iota(X)$, whence $\iota(X) g^{(1)}$ is simple. That is, the element $\alpha=b_{1} \ldots b_{s} g^{(1)}$ is simple.

By the above commutative diagram, we know that $\alpha=b_{1} \ldots b_{s} g^{(1)}=g_{0} b_{1}^{\prime} \ldots b_{s}^{\prime}$, where $g_{0}$ is a grey arrow and $\left(b_{1}^{\prime}, \ldots, b_{s}^{\prime}\right)$ is a black path. Hence, by Corollary 3.22, $g_{0}$ and $b_{1}^{\prime} \ldots b_{s}^{\prime}$ are determined by $\alpha$. More precisely, the left normal form of $\varphi(X) \alpha$ is equal to $\left(\varphi(X) g_{0}\right)\left(b_{1}^{\prime} \ldots b_{s}^{\prime}\right)$.

On the other hand, we know by [18, Lemma 2.3] that $\alpha=\iota(X) g^{(1)}=g \iota(T)$. Since $\iota(T)$ is an element that conjugates $T$ to $\mathbf{c}(T)$ and since $T, \mathbf{c}(T) \in \operatorname{USS}(X)$, the element $\iota(T)$ can be decomposed as a product of minimal simple elements $\iota(T)=$ 
$b_{1}^{\prime \prime} \ldots b_{t}^{\prime \prime}$. Moreover, $\left(b_{1}^{\prime \prime}, \ldots, b_{t}^{\prime \prime}\right)$ is an oriented path starting at $T$, whose associated conjugating element is $\iota(T)$. By Corollary $3.16,\left(b_{1}^{\prime \prime}, \ldots, b_{t}^{\prime \prime}\right)$ is a black path. Hence, $\left(g, b_{1}^{\prime \prime}, \ldots, b_{t}^{\prime \prime}\right)$ is a grey-black path, whose associated conjugating element is $\alpha$. By Corollary 3.22, the left normal form of $\varphi(X) \alpha$ is equal to $(\varphi(X) g)\left(b_{1}^{\prime \prime} \ldots b_{t}^{\prime \prime}\right)$. Therefore $g_{0}=g$ and $b_{1}^{\prime} \ldots b_{s}^{\prime}=b_{1}^{\prime \prime} \ldots b_{t}^{\prime \prime}=\iota(T)$, as we wanted to show.

Consider the first square of the above commutative diagram:

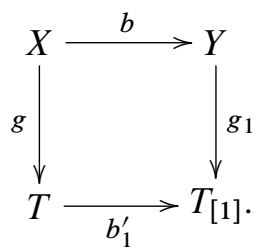

We want to define $g_{1}$ as being the partial transport of $g$ along $b$. But this will only be consistent if $g_{1}$ is completely determined by $X, b$ and $g$ (and does not depend on the decomposition $c=b_{2} \ldots b_{s}$ chosen). We will see that this is the case. Indeed, $g^{(1)}$ is completely determined by $X$ and $g$, while $c$ is determined by $X$ and $b$. Hence $c g^{(1)}$ is determined by $X, b$ and $g$. We then use a decomposition $c g^{(1)}=g_{1} b_{2}^{\prime} \ldots b_{s}^{\prime}$, where $\left(g_{1}, b_{2}^{\prime}, \ldots, b_{s}^{\prime}\right)$ is a grey-black path starting at $Y$. By Corollary 3.22 , the grey part of this path, that is $g_{1}$, is determined by $c g^{(1)}$ and $Y=X^{b}$, hence it is determined by $X, b$ and $g$, as we wanted to show. More specifically, Corollary 3.22 yields $g_{1}=\partial(\varphi(Y)) \wedge c g^{(1)}$.

Recalling $b c=\iota(X)$ and $\iota(X) g^{(1)}=g \iota(T)$ [18, Lemma 2.3], we can rewrite this as

$g_{1}=\partial(\varphi(Y)) \wedge\left(b^{-1} \iota(X) g^{(1)}\right)=\partial(\varphi(Y)) \wedge\left(b^{-1} g \iota(T)\right)=\partial\left(\varphi\left(X^{b}\right)\right) \wedge\left(b^{-1} g \iota\left(X^{g}\right)\right)$.

In particular, this expression for $g_{1}$ depends only on $X, b$ and $g$.

Definition 3.27. Let $X \in G$ be rigid and $\ell(X)>1$. Let $b$ and $g$ be arrows in $\Gamma_{X}$ starting at $X$, where $b$ is black and $g$ is grey, and let $Y=X^{b}$ and $T=X^{g}$. Then we define the partial transport of $g$ along $b$ as follows:

$$
g^{[b]}=\partial(\varphi(Y)) \wedge\left(b^{-1} g \iota(T)\right) .
$$

Before showing some properties of this partial transport, it will be helpful to also define the partial transport of a black arrow along a grey one. Using the analogy between grey and black arrows, considering the graph $\Gamma_{X^{-1}}$, we just need to recall that $\partial\left(\varphi\left(T^{-1}\right)\right)=\iota(T)$ and $\iota\left(Y^{-1}\right)=\partial(\varphi(Y))$. Hence we have

Definition 3.28. Let $X \in G$ be rigid and $\ell(X)>1$. Let $b$ and $g$ be arrows in $\Gamma_{X}$ starting at $X$, where $b$ is black and $g$ is grey, and let $Y=X^{b}$ and $T=X^{g}$. Then we define the partial transport of $b$ along $g$ as follows:

$$
b^{[g]}=\iota(T) \wedge\left(g^{-1} b \partial(\varphi(Y))\right) .
$$


Fortunately, the partial transport $b^{[g]}$ is precisely the element $b_{1}^{\prime}$ in the above commutative diagram. This is shown in the following result:

Lemma 3.29. In the above situation, the following diagram is commutative:

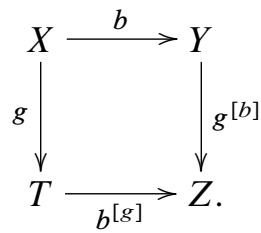

Proof. Since $g^{[b]}=\partial(\varphi(Y)) \wedge\left(b^{-1} g \iota(T)\right)$, one has $b g^{[b]}=b \partial(\varphi(Y)) \wedge g \iota(T)$. On the other hand, since $b^{[g]}=\iota(T) \wedge\left(g^{-1} b \partial(\varphi(Y))\right)$, one has $g b^{[g]}=g \iota(T) \wedge$ $b \partial(\varphi(Y))$. Therefore, $b g^{[b]}=g b^{[g]}$, as we wanted to show.

Remark. Looking at the above diagram, one may be tempted to conjecture that $b g^{[b]}=g b^{[g]}=b \vee g$. This, however, is not true in general. A counterexample in the braid group $B_{4}$ is given by the rigid braid $X=\left(\sigma_{2}\right)\left(\sigma_{2} \sigma_{1} \sigma_{3} \sigma_{2}\right)\left(\sigma_{2} \sigma_{1} \sigma_{3}\right)\left(\sigma_{1} \sigma_{2}\right), g=$ $\sigma_{1}, b=\sigma_{2}$. In this case $b \vee g=\sigma_{1} \sigma_{2} \sigma_{1}$ and $X^{b \vee g}=\left(\sigma_{1} \sigma_{3} \sigma_{2}\right)\left(\sigma_{2} \sigma_{1} \sigma_{3} \sigma_{2}\right)\left(\sigma_{2}\right)\left(\sigma_{2} \sigma_{1}\right)$ which is not rigid, that is, does not belong to $\operatorname{USS}(X)$. Since $g b^{[g]}$ conjugates $X$ to an element in $\operatorname{USS}(X)$, it follows that $b \vee g \neq g b^{[g]}$. More precisely, in this case $b^{[g]}=\sigma_{2} \sigma_{1} \sigma_{3}$, hence $g b^{[g]}=\sigma_{1} \sigma_{2} \sigma_{1} \sigma_{3} \neq \sigma_{1} \sigma_{2} \sigma_{1}=b \vee g$.

We will now show some properties of the partial transport which shall provide more information about the structure of $\Gamma_{X}$. First, let us see that the diagram (4) is the only possible commutative diagram that completes the diagram

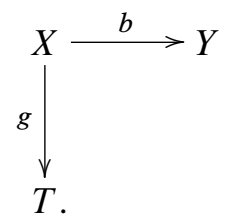

Proposition 3.30. Let $X \in G$ be rigid and $\ell(X)>1$. Let $b$ be a black arrow in $\Gamma_{X}$ starting at $X$ and ending at $Y$ and let $g$ be a grey arrow starting at $X$ and ending at $T$. Suppose that $b^{\prime}$ is a black arrow starting at $T$ and $g^{\prime}$ is a grey arrow starting at $Y$, such that $b g^{\prime}=g b^{\prime}$. Then $b^{\prime}=b^{[g]}$ and $g^{\prime}=g^{[b]}$.

Proof. By definition, we have $b g^{[b]}=b \partial(\varphi(Y)) \wedge g \iota(T)$. However, since $g^{\prime}$ is a grey arrow starting at $Y$ one has $g^{\prime} \preccurlyeq \partial(\varphi(Y))$ so $b g^{\prime} \preccurlyeq b \partial(\varphi(Y))$. Similarly, since $b^{\prime}$ is a black arrow starting at $T$, one has $b^{\prime} \preccurlyeq \iota(T)$, hence $b g^{\prime}=g b^{\prime} \preccurlyeq g \iota(T)$. Therefore, $b g^{\prime} \preccurlyeq b g^{[b]}$, that is, $g^{\prime} \preccurlyeq g^{[b]}$. Since both arrows correspond to minimal simple elements, they must be equal, that is, $g^{\prime}=g^{[b]}$.

Finally, one has $g b^{\prime}=b g^{\prime}=b g^{[b]}=g b^{[g]}$, hence $b^{\prime}=b^{[g]}$. 
Corollary 3.31. Let $X \in G$ be rigid and $\ell(X)>1$. Let $b$ be a black arrow in $\Gamma_{X}$ starting at $X$ and ending at $Y$. Then the partial transport along $b$ is a bijective map from the set of grey arrows starting at $X$ to the set of grey arrows starting at $Y$.

Proof. Suppose that $g_{1}$ and $g_{2}$ are two grey arrows starting at $X$ such that $g_{1}^{[b]}=g_{2}^{[b]}$. Then $g_{1} b^{\left[g_{1}\right]}=b g_{1}^{[b]}=b g_{2}^{[b]}=g_{2} b^{\left[g_{2}\right]}$. Hence, $\left(g_{1}, b^{\left[g_{1}\right]}\right)$ and $\left(g_{2}, b^{\left[g_{2}\right]}\right)$ are two grey-black paths starting at $X$ and having the same associated element. By Corollary 3.22, the grey parts of these paths coincide, so $g_{1}=g_{2}$. Hence, the partial transport along $b$ is injective.

Now let $g^{\prime}$ be a grey arrow starting at $Y$. Then $\left(b, g^{\prime}\right)$ is an oriented path starting at $X$ formed by a black arrow followed by a grey arrow. By Proposition 3.23, there exist a unique grey arrow $g$ and a unique black arrow $b^{\prime}$ such that $\left(g, b^{\prime}\right)$ is an oriented path starting at $X$ and $b g^{\prime}=g b^{\prime}$. By the above result, one necessarily has $g^{\prime}=g^{[b]}$. Hence the partial transport along $b$ is surjective.

Corollary 3.32. Let $X \in G$ be rigid and $\ell(X)>1$. Let $g$ be a grey arrow in $\Gamma_{X}$ starting at $X$ and ending at $T$. Then the partial transport along $g$ is a bijective map from the set of black arrows starting at $X$ to the set of black arrows starting at $T$.

Proof. This result is equivalent to the previous one, if we replace $X$ by $X^{-1}$.

Corollary 3.33. Let $X \in G$ be rigid and $\ell(X)>1$. Then every two elements in a black component of $\Gamma_{X}$ admit the same number of grey arrows and every two elements in a grey component of $\Gamma_{X}$ admit the same number of black arrows.

Proof. If two elements $Y$ and $Z$ belong to the same black component of $\Gamma_{X}$, then they can be connected by a sequence of black arrows. By Corollary 3.31, the partial transport along each one of these black arrows is a bijection. Hence, the composition of all these partial transports is a bijection from the set of grey arrows starting at $Y$ to the set of grey arrows starting at $Z$. An analogous proof shows the same statement with reversed colors.

In order to complete the picture, we will show that this definition of partial transport is consistent with the transport defined in [18]. This is done by the following two results.

Lemma 3.34. Let $X, Y, T, b$ and $g$ as above.

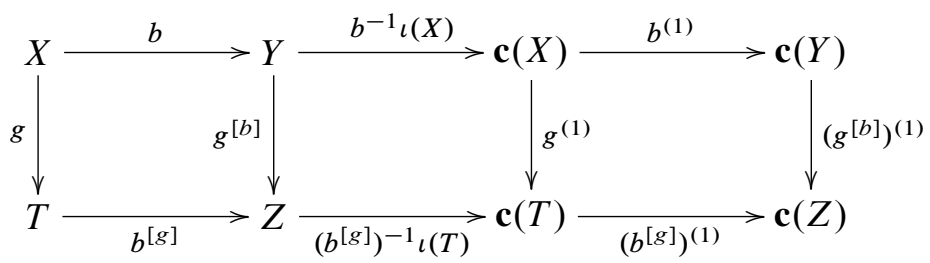


(1) The transport of $g^{[b]}$ is equal to the partial transport of $g^{(1)}$ along $b^{(1)}$. That is, $\left(g^{[b]}\right)^{(1)}=\left(g^{(1)}\right)^{\left[b^{(1)}\right]}$.

(2) The transport of $b^{[g]}$ is equal to the partial transport of $b^{(1)}$ along $g^{(1)}$. That is, $\left(b^{[g]}\right)^{(1)}=\left(b^{(1)}\right)^{\left[g^{(1)}\right]}$.

Proof. By [18, Corollary 2.2] the transport is multiplicative, whence $\left(b g^{[b]}\right)^{(1)}=$ $b^{(1)}\left(g^{[b]}\right)^{(1)}$. However, since $b g^{[b]}=g b^{[g]}$, one also has $\left(b g^{[b]}\right)^{(1)}=\left(g b^{[g]}\right)^{(1)}=$ $g^{(1)}\left(b^{[g]}\right)^{(1)}$. Hence, $b^{(1)}\left(g^{[b]}\right)^{(1)}=g^{(1)}\left(b^{[g]}\right)^{(1)}$. In other words, if we consider the subdiagrams
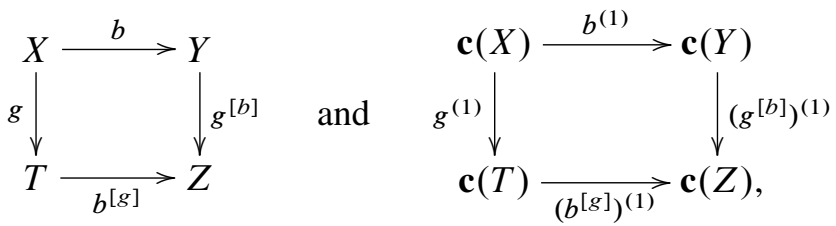

then the commutativity of the first one implies the commutativity of the second one.

The transport is monotonic with respect to $\preccurlyeq$ by [18, Corollary 2.2], whence $b \preccurlyeq \iota(X)$ implies $b^{(1)} \preccurlyeq \iota(X)^{(1)}=\iota(\mathbf{c}(X))$. That is, the transport sends black arrows to black arrows. As the transport is a bijection on the set of all arrows and the set of grey arrows is the complement of the set of black arrows as $X$ is rigid, this means that the transport sends grey arrows to grey arrows. In particular, $b^{(1)}$ and $\left(b^{[g]}\right)^{(1)}$ are black arrows and $g^{(1)}$ and $\left(g^{[b]}\right)^{(1)}$ are grey ones. By Proposition 3.30 and the commutativity of the second diagram above, this implies $\left(g^{[b]}\right)^{(1)}=\left(g^{(1)}\right)^{\left[b^{(1)}\right]}$ and $\left(b^{[g]}\right)^{(1)}=\left(b^{(1)}\right)^{\left[g^{(1)}\right]}$, as we wanted to show.

We will finally show that several partial transports lead to a full transport. Recall the commutative diagram

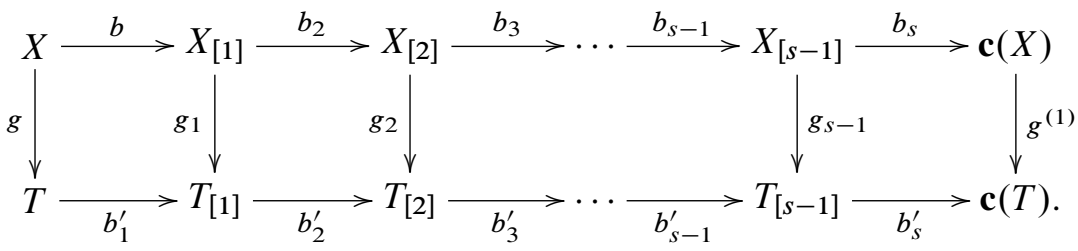

Recall that $g_{1}=g^{[b]}$ and $b_{1}^{\prime}=b^{[g]}$. Denote $g^{\left[b_{1}, b_{2}, \ldots, b_{i}\right]}=\left(\ldots\left(\left(g^{\left[b_{1}\right]}\right)^{\left[b_{2}\right]}\right) \ldots\right)^{\left[b_{i}\right]}$, where $b_{1}=b$. Denote also $g_{s}=g^{(1)}$.

Lemma 3.35. With the above notations, $g^{\left[b_{1}, \ldots, b_{i}\right]}=g_{i}$ for $i=1, \ldots s$. In particular, $g^{\left[b_{1}, \ldots, b_{s}\right]}=g^{(1)}$. 
Proof. We just need to notice that, by construction, each square in the above diagram is commutative, and is made of black and grey arrows in the appropriate sense. Hence by Proposition $3.30, g_{i}=g_{i-1}^{\left[b_{i}\right]}$ for $i=2, \ldots, s$, so the result holds.

Therefore, the transport defined in [18] can be seen in the rigid case as an iterated partial transport along a black path whose associated conjugating element is $\iota(X)$. We have thus seen how special the structure of $\Gamma_{X}$ is when $X$ is a rigid element with $\ell(X)>1$.

\section{Periodic elements}

After the study of rigid elements, we will study other kind of elements in a Garside group whose associated ultra summit graph is, in some sense, in the other extreme. This is the case of periodic elements.

Definition 4.1. An element $X \in G$ is said to be periodic if $X^{m}=\Delta^{k}$ for some nonzero integers $m$ and $k$.

Example 4.2. The 5-braid $C$ that we gave earlier, in Example 2.13, is a periodic braid. We shall see soon that its graph $\Gamma_{C}$, depicted in the left sketch in Figure 3, illustrates many of the results in this section.

In this section we will study the structure of the ultra summit set of a periodic element $X$. We know that if $X$ were rigid and $\ell(X)>1$, then no arrow in $\Gamma_{X}$ would be bi-colored. The case of periodic elements is completely the opposite: we will show that if $X$ is periodic and $\ell(X)>0$, then every arrow in $\Gamma_{X}$ is bi-colored. Hence $\Gamma_{X}=\mathscr{B}_{X}=\mathscr{E}_{X}$. In particular, every two elements in $\operatorname{USS}(X)$ can be connected by a sequence of partial cyclings.

Theorem 4.3 ([2, Theorem 4.5] for Artin-Tits groups, [9, Corollary 6.9] for Garside groups)). If $X \in \operatorname{SSS}(X)$ is periodic and it is not a power of $\Delta$, then $\ell(X)=1$.

Remark 4.4. A new proof of Theorem 4.3 for all Garside groups follows immediately from the decomposition that is given in [3, Theorem 2.9].

Recall that an atom is an indivisible simple element, and that every Garside group $G$ is generated by its atoms. We will show that if an element $X \in \operatorname{USS}(X)$ is periodic, then all its minimal simple elements are atoms and correspond to partial cyclings. We will also determine which atoms are minimal simple elements for a given periodic element.

Here is the main result concerning periodic elements. 
Proposition 4.5. Let $X \in \operatorname{USS}(X)$ be a periodic element which is not a power of $\Delta$. The set of minimal simple elements for $X$ is equal to the set of atoms dividing $\iota(X)$ and $\iota\left(X^{-1}\right)$, that is, the left descent set of $\iota(X) \wedge \iota\left(X^{-1}\right)$, in the terminology of Artin-Tits groups.

Proof. For every $Y \in G, \operatorname{SSS}\left(Y^{-1}\right)$ consists of the inverses of the elements in $\operatorname{SSS}(Y)$; see Remark 1.8. In our case, since $X$ is periodic and it is not a power of $\Delta$, we have $\ell(X)=1$. Hence, $\operatorname{USS}(X)=\operatorname{SSS}(X)$ and $\operatorname{USS}\left(X^{-1}\right)=\operatorname{SSS}\left(X^{-1}\right)=$ $\left\{Y^{-1} \mid Y \in \operatorname{SSS}(X)\right\}$ by Lemma 3.7. Moreover, a simple element $t$ satisfies $X^{t} \in$ $\operatorname{USS}(X)$, that is $\ell\left(t^{-1} X t\right)=1$, if and only if $\ell\left(t^{-1} X^{-1} t\right)=1$, which is equivalent to $\left(X^{-1}\right)^{t} \in \operatorname{USS}\left(X^{-1}\right)$. Therefore, the minimal simple elements for $X$ and for $X^{-1}$ coincide.

Let $\Delta^{p} x_{1}$ be the left normal form of $X$, and recall that $\iota(X)=\tau^{-p}\left(x_{1}\right)$ and $\iota\left(X^{-1}\right)=\partial\left(x_{1}\right)$. Let $s$ be a minimal simple element for $X$ (thus for $X^{-1}$ ). By Corollary 2.7, we have $s \preccurlyeq \iota(X)$ or $s \preccurlyeq \iota\left(X^{-1}\right)$. Replacing $X$ by $X^{-1}$ if necessary, we can assume that $s \preccurlyeq \iota\left(X^{-1}\right)=\partial\left(x_{1}\right)$. This implies that $x_{1} s$ is simple.

We will show that we also have $s \leqslant \iota(X)=\tau^{-p}\left(x_{1}\right)$ or, equivalently, that $\tau^{p}(s) \preccurlyeq x_{1}$. We know that $\tau^{p}(s) \vee x_{1}=x_{1} t$, for some simple element $t$. We must then show that $t=1$.

Notice that $\ell\left(s^{-1} X s\right)=\ell\left(s^{-1} \Delta^{p} x_{1} s\right)=1$, hence $\tau^{p}(s)^{-1} x_{1} s$ is simple. That is, $\tau^{p}(s) \preccurlyeq x_{1} s$. Then, $x_{1} s$ is a multiple of $\tau^{p}(s)$ and of $x_{1}$, so it follows that $x_{1} t \preccurlyeq x_{1} s$, thus $t \preccurlyeq s$. This yields $\tau^{p}(t) \preccurlyeq \tau^{p}(s) \preccurlyeq x_{1} t$, whence $\tau^{p}(t)^{-1} x_{1} t$ is simple, implying $\ell\left(t^{-1} X t\right)=1$. By minimality of $s$, it follows that either $t=1$ (as we want to show) or $t=s$.

Suppose that $t=s$, that is, $\tau^{p}(s) \vee x_{1}=x_{1} s$. We will see that in this case $X$ cannot be periodic, since we will show by induction that $\ell\left(X^{k}\right)>0$ and that $s \nless \iota\left(X^{k}\right)$, for every $k>0$. For $k=1$, we know that $\ell(X)=1$, and if we had $s \preccurlyeq \iota(X)=\tau^{-p}\left(x_{1}\right)$, then $\tau^{p}(s) \preccurlyeq x_{1}$, so $\tau^{p}(s) \vee x_{1}=x_{1}=x_{1} s$, which would imply that $s=1$ in contradiction to our assumption. Hence, the claim is true for $k=1$.

Suppose the claim is true for some $k \geq 1$. Let $\Delta^{q} z_{1} \ldots z_{m}$ be the left normal form of $X^{k}$, where $m \geq 1$. Then $X^{k+1}=\Delta^{p} x_{1} \Delta^{q} z_{1} \ldots z_{m}$. In order to show that $\ell\left(X^{k+1}\right)>0$ it suffices to prove that $x_{1} \tau^{-q}\left(z_{1}\right)$ is not a multiple of $\Delta$. This follows if we can show that $\partial\left(x_{1}\right) \nless \tau^{-q}\left(z_{1}\right)$, that is, that $\iota\left(X^{-1}\right) \nless \iota\left(X^{k}\right)$. The latter is true since we are assuming that $s \preccurlyeq \iota\left(X^{-1}\right)$, while by induction hypothesis $s \nless \iota\left(X^{k}\right)$. Hence $\ell\left(X^{k+1}\right)>0$. Moreover, in this case $\iota\left(X^{k+1}\right)$ is the maximal simple prefix of $\tau^{-p}\left(x_{1}\right) \tau^{-p-q}\left(z_{1}\right)$. If we had $s \preccurlyeq \tau^{-p}\left(x_{1}\right) \tau^{-p-q}\left(z_{1}\right)$, then $\tau^{p}(s) \preccurlyeq x_{1} \tau^{-q}\left(z_{1}\right)=$ $x_{1} \iota\left(X^{k}\right)$. In this case, $x_{1} \iota\left(X^{k}\right)$ would be a multiple of $\tau^{p}(s)$ and of $x_{1}$, whence we would have $x_{1} s \preccurlyeq x_{1} \iota\left(X^{k}\right)$ as we assume $\tau^{p}(s) \vee x_{1}=x_{1} s$. This is not possible, since by induction hypothesis $s \nless \iota\left(X^{k}\right)$. Therefore, $s \nless \tau^{-p}\left(x_{1}\right) \tau^{-p-q}\left(z_{1}\right)$, whence $s \notin \iota\left(X^{k+1}\right)$ which proves the claim.

We have then shown that if $t=s$, the element $X$ would not be periodic. Hence, 
we have $t=1$. This means that $\tau^{p}(s) \vee x_{1}=x_{1}$, that is, $\tau^{p}(s) \preccurlyeq x_{1}$, which implies that $s \preccurlyeq \tau^{-p}\left(x_{1}\right)=\iota(X)$. Therefore, every minimal simple element $s$ for $X$ must be a prefix of $\iota\left(X^{-1}\right)$ and also of $\iota(X)$.

Now choose any atom $a \preccurlyeq s$. We know that $a \preccurlyeq \iota(X)=\tau^{-p}\left(x_{1}\right)$ and that $x_{1} a$ is simple. Therefore, $a^{-1} X a$ has canonical length 1 . By minimality of $s$, we have $a=s$. Hence, every minimal simple element for $X$ is an atom dividing both $\iota(X)$ and $\iota\left(X^{-1}\right)$.

Conversely, an atom $a$ which divides both $\iota(X)$ and $\iota\left(X^{-1}\right)$ satisfies $\tau^{p}(a) \preccurlyeq x_{1}$ and $x_{1} a$ is simple, whence $\ell\left(a^{-1} X a\right)=1$. Since an atom has no proper prefixes, it is a minimal simple element for $X$.

The most important consequence of this result is that one can connect every two elements in the ultra summit set of a periodic element by a sequence of partial cyclings.

Corollary 4.6. Let $X \in \operatorname{USS}(X)$ and $Y \in \operatorname{USS}(Y)$ be periodic elements. Then $X$ and $Y$ are conjugate if and only if there exists a sequence of elements $X=$ $X_{1}, X_{2}, \ldots, X_{m}=Y$, such that for all $i=1, \ldots, m-1$, the element $X_{i+1}$ is $a$ partial cycling of $X_{i}$ and $X_{i} \in \operatorname{USS}\left(X_{i}\right)=\operatorname{USS}(X)$.

Proof. If $X=\Delta^{m}$ for some $m$, then $\operatorname{USS}(X)=\{X\}$, so the result is trivially true. We can then assume that $X$ is not a power of $\Delta$.

Suppose that $X$ and $Y$ are conjugate. By [18, Corollary 1.19] and Remark 1.11 there exists a chain of elements $X=X_{1}, X_{2}, \ldots, X_{m}=Y$, such that $X_{i+1}=X_{i}^{s_{i}}$, where $X_{i} \in \operatorname{USS}(X)$ and $s_{i}$ is a minimal simple element for $X_{i}$, for $i=1, \ldots, m-1$. By Proposition 4.5 , each $s_{i}$ is an atom dividing $\iota\left(X_{i}\right)$, whence $X_{i+1}$ is a partial cycling of $X_{i}$, for $i=1, \ldots, m-1$.

The converse is trivial.

We will see in Proposition 5.4 that, given $X \in G$, we can obtain some $X^{\prime} \in$ $\mathrm{USS}(X)$ by a finite number of partial cyclings. Given $X, Y \in G$ which are conjugate, we can hence obtain $X^{\prime} \in \operatorname{USS}(X)$ and $Y^{\prime} \in \operatorname{USS}(X)$ using partial cyclings. If $X$ is periodic, we have also shown that one can go from $X^{\prime}$ to $Y^{\prime}$ by a finite number of partial cyclings.

But in general, even if $X$ is periodic, one cannot go from $X$ to $Y$ by partial cyclings, since a partial cycling will never decrease the infimum of an element, and $Y$ does not necessarily have maximal infimum.

\section{Applications}

5.1. Complexity of the CDP/CSP for pseudo-Anosov and periodic braids. The work in this paper is applicable to all Garside groups, and no assumptions are made that restrict attention to the braid groups $B_{n}$. In this section we show, by applying our 
results to braids, that we have made progress toward one of our long-range goals. That goal is to find a solution to the CDP/CSP for PA elements in $B_{n}$ which is polynomial, that is bounded above by a polynomial in both $n$ and $\ell(X)$. In this section we discuss our progress, at this time, toward that goal, and what remains to be done.

Putting $X$ into left normal form is known to have polynomial complexity; see [15, Corollary 9.5.3] and [5, Theorem 4.4]. It follows from [6, Theorem 1] that there is a polynomial bound to how many times one must cycle and decycle an element $X$ in order to bring $X$ to a representative which is in $\operatorname{SSS}(X)$, so without loss of generality we may assume that $X$ is in left normal form and in $\operatorname{SSS}(X)$. But at this time we do not know how many times one must cycle to bring $X$ into $\operatorname{USS}(X)$. That problem may yield, using the machinery developed in $\$ 2$ of [3], however at this writing it remains an open question. Let us assume from now on that $X \in \operatorname{USS}(X)$.

(A) The PA case. We have proved in [3, Theorem 3.37] that, if $X$ is PA, then there is an integer $m$ which is at most $((n)(n-1) / 2)^{3}$, such that every element in $\operatorname{USS}\left(X^{m}\right)$ is rigid if we use the usual Garside structure. If we use the BKL structure [5] then $m$ is bounded by $(n-1)^{3}$. In view of the fact, proved in $[19, \S 4.2]$, that roots of PA braids are unique, this means that, in searching for a polynomial solution to the CDP/CSP for PA braids, we may assume that they are rigid. Since any rigid element $X$ of a Garside group is in $\operatorname{USS}(X)$, this means we may assume that $X$ is PA and that $X \in \operatorname{USS}(X)$ is rigid.

We have computed many many examples of $\operatorname{USS}(X)$ when $X$ is PA and rigid, using random searches, and on the basis of the evidence found that in the generic case $\operatorname{USS}(X)$ has either 2 orbits, where one is the conjugate of the other by $\Delta$, or 1 orbit which is conjugate to itself by $\Delta$. Those are the two cases that we saw in Example 2.11 and Figure 1. We found this behavior over and over again, in calculations with very large numbers of randomly chosen examples, when we restricted our attention to PA braids that are rigid. A question that remains, for future work, is: If $X$ is a PA and rigid element in $B_{n}$, is the size of $\operatorname{USS}(X)$ bounded above by some polynomial in $n$ and $\ell(X)$ ? This is where our structure theorems about $\operatorname{USS}(X)$ become very important. We present two examples which illustrate the problems that remain to be solved in the PA, rigid case. Let us remember that in such cases we understand cycling very well, but the partial cyclings and partial twisted decyclings that connect cycling orbits present new combinatorial challenges.

We would like to give some examples to illustrate the difficulties, but encounter a problem on how to both present interesting examples and draw good pictures, when $\operatorname{USS}(X)$ is unexpectedly large. To overcome the difficulty, recall that for every $X \in G$ there is an automorphism $\phi$ of $\Gamma_{X}$ that sends every vertex $Y$ to $\mathbf{c}(Y)$ and every arrow $s$ to its transport $s^{(1)}$. We can hence define a quotient graph $\Gamma_{X} / \phi$ whose vertices are the cycling orbits in the vertex set of $\Gamma_{X}$. Recall also that $\phi$ preserves the colors of the arrows, hence the arrows in $\Gamma_{X} / \phi$ also have a well-defined color (black or grey). We can then draw the quotient graphs $\Gamma_{X} / \phi$ to have an idea how the distinct orbits 
are connected in the graph $\Gamma_{X}$. In the two quotient graphs, Figures 4 and 5 below, the vertex label $E_{i}$ or $F_{i}$ means a cycling orbit.

Example 5.1. Figure 4 illustrates one of the difficulties using the 12-braid $E$ :

$$
\begin{aligned}
E=\left(\sigma_{2} \sigma_{1} \sigma_{7} \sigma_{6} \sigma_{5} \sigma_{4} \sigma_{3} \sigma_{8} \sigma_{7} \sigma_{11} \sigma_{10}\right) \cdot\left(\sigma_{1} \sigma_{2} \sigma_{3} \sigma_{2} \sigma_{1} \sigma_{4} \sigma_{3} \sigma_{10}\right) \\
\cdot\left(\sigma_{1} \sigma_{3} \sigma_{4} \sigma_{10}\right) \cdot\left(\sigma_{1} \sigma_{10}\right) \cdot\left(\sigma_{1} \sigma_{10} \sigma_{9} \sigma_{8} \sigma_{7} \sigma_{11}\right) \cdot\left(\sigma_{1} \sigma_{2} \sigma_{7} \sigma_{11}\right)
\end{aligned}
$$

The braid $E$ is a PA, rigid braid, with cycling orbits of length 6. It turns out that $\Gamma_{E}$ has 264 elements, so the quotient graph $\Gamma_{E} / \phi$ has 44 vertices. Of course we cannot distinguish between elements in the same cycling orbit in the quotient graph, however with some extra information we will be able to understand $\Gamma_{E}$ too:

(1) There is a black arrow from $E_{i, j}$ to $E_{i+2, j}$ for every $i \neq 43,44$.

(2) There is a black arrow from $E_{i, j}$ to $E_{i-2, j+1}$ for every $i \neq 1,2$.

(3) The product of two consecutive black arrows in opposite senses is trivial on orbits, but (in this example) corresponds to cycling of the elements in the orbit.

(4) There is a grey arrow from $E_{i, j}$ to $E_{i+1, j-1}$ if $i$ is odd.

(5) There is a grey arrow from $E_{i, j}$ to $E_{i-1, j-1}$ if $i$ is even.

(6) Every grey arrow corresponds to a twisted decycling. (Hence $\tau\left(E_{i}\right)=E_{i+1}$ for every odd $i$ ).

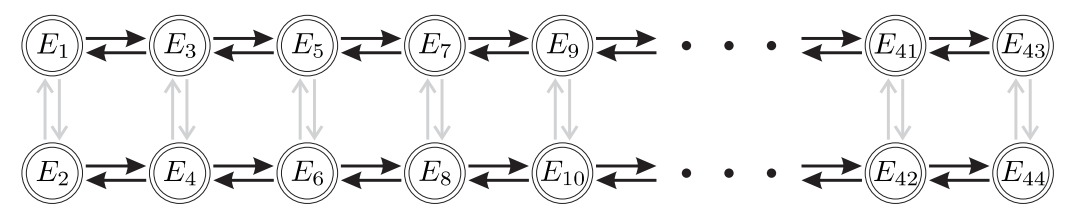

Figure 4. The quotient graph $\Gamma_{E} / \phi$ illustrates a very long path of iterated partial cyclings.

Example 5.2. Figure 5 illustrates another way in which partial cycling leads to difficult combinatorial problems. Consider the 12-braid $F$ :

$$
\begin{aligned}
F=( & \left(\sigma_{3} \sigma_{2} \sigma_{1} \sigma_{4} \sigma_{6} \sigma_{8} \sigma_{7} \sigma_{6} \sigma_{9} \sigma_{10} \sigma_{11} \sigma_{10}\right) \cdot\left(\sigma_{1} \sigma_{2} \sigma_{4} \sigma_{3} \sigma_{2} \sigma_{1} \sigma_{5} \sigma_{7} \sigma_{10} \sigma_{11} \sigma_{10}\right) \\
\cdot & \cdot\left(\sigma_{3} \sigma_{5} \sigma_{7} \sigma_{10} \sigma_{11} \sigma_{10}\right) \cdot\left(\sigma_{3} \sigma_{5} \sigma_{7} \sigma_{6} \sigma_{8} \sigma_{10} \sigma_{11}\right)
\end{aligned}
$$

Since $F$ is rigid and since $\ell(F)=4$ each cycling orbit has 4 elements. The graph $\Gamma_{F}$ has two isomorphic black components, each made of 29 cycling orbits. We show one of them. Its graph has the following properties, the key one being Property (3): 


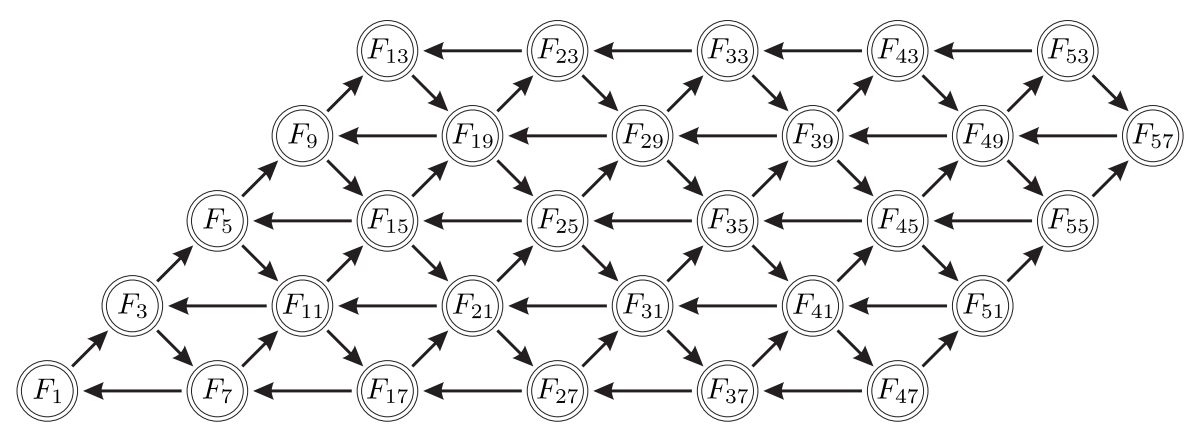

Figure 5. The quotient graph $\Gamma_{F} / \phi$ illustrates multiple minimal partial cyclings at a vertex.

(1) Every black arrow pointing toward the right (joining orbits $i$ and $j$ ) goes from $F_{i, k}$ to $F_{j, k}$.

(2) Every black arrow pointing to the left (joining orbits $i$ and $j$ ) goes from $F_{i, k}$ to $F_{j, k+1}$.

(3) The concatenation of three black arrows forming a 'small triangle' corresponds to a cycling. Hence, by Lemma 3.1, the initial factor of every vertex of $\Gamma_{F}$ which is not in $F_{1}$ can be decomposed in several ways as a product of minimal simple elements. For example, the element $F_{11,1}$ has 6 different decompositions of $\iota\left(F_{11,1}\right)$, given by the small triangles $11-3-7,11-3-5,11-15-5,11-15-21,11-$ 17-21, 11-17-7. This causes 'branching' at the vertex $F_{11,1}$.

(4) We have grey arrows, corresponding to partial twisted decyclings, going from $F_{2 k-1, j}$ to $F_{2 k, j-1}$ and also from $F_{2 k, j}$ to $F_{2 k-1, j-1}$.

Here are the problems that remain, with regard to PA braids. First, we need to learn how many times we must cycle to bring $X \in \operatorname{SSS}(X)$ into a closed cycling orbit, i.e. into USS $(X)$. This is 'Open question 3 ' in [3, $\S 1.4]$. We have shown in [3, Theorem 3.37] that once we are in $\operatorname{USS}(X)$, we may assume that $X$ is rigid. If $X$ is in left normal form and is rigid, then the length of every cycling orbit is either $\ell(X)$ or $2 \ell(X)$. Lemma 3.1 shows that very very special combinatorial conditions are required for partial cycling, and even more for iterated partial cycling, to occur. Yet Examples $E$ and $F$ show that $\operatorname{USS}(X)$ can have surprises. We must find polynomial bounds on (i) the lengths of paths in $\Gamma_{X}$ and (ii) the number of such paths (that is, the combinatorics introduced by branching). For fixed braid index and length $\ell(X)$ we need a universal bound on the lengths and numbers of such paths. If we can solve all these problems, then we should be able to solve 'Open question 2' of $\$ 1.4$ of [3], that is to find a polynomial bound on $|\operatorname{USS}(X)|$ and so (using all our other work in [3] and this paper, as well as drawing heavily on the literature), obtain a polynomial solution to the CDP/CSP for PA braids. 
(B) The periodic case. The situation for periodic braids is quite different from that for PA braids. On the one hand, periodic braids are quite simple. It is well-known from the work of Eilenberg [13] and de Kerékjártó [20] that every periodic element $X \in B_{n}$ is conjugate to a rotation which is either a power of $\delta=\sigma_{n-1} \sigma_{n-2} \ldots \sigma_{1}$ or a power of $\varepsilon=\delta \sigma_{1}$; see [10, Theorem 3.1] for a modern exposition of this work. However, unfortunately, it turns out that $|\operatorname{USS}(\delta)|=2^{n-2}$ and $|\operatorname{USS}(\varepsilon)|=(n-2) 2^{n-3}$ (this will be proved in [4]), so without even considering powers of $\delta$ and $\varepsilon$ we have exponential growth. We will nevertheless arrive at a polynomial solution to the CDP/CSP in [4], by introducing new tricks that put the two known Garside structures on $B_{n}$ to work, and make use of Garside structures on other Artin groups too. That is the content of the manuscript [4].

5.2. A new solution to the CDP/CSP in Garside groups. In this section, we use earlier results to present a new solution of the CDP/CSP problem in a Garside group $G$, that is, we give an algorithm which determines whether two elements $X, Y \in G$ are conjugate. Moreover, if $X$ and $Y$ are conjugate, the algorithm finds a conjugating element $\alpha$ such that $X^{\alpha}=Y$.

Recall that the algorithm in [18] is based on computing $\operatorname{USS}(X)$ and one element $Y^{\prime} \in \operatorname{USS}(Y)$. Then, $X$ and $Y$ are conjugate if and only if $Y^{\prime} \in \operatorname{USS}(X)$. Hence, if one uses the algorithm in [18], one must in the worst case compute the entire set USS $(X)$. Corollary 2.26, however, shows that this can be avoided. Here is the promised algorithm. It is based upon Corollary 2.27

\section{Algorithm 3}

Input: $X, Y \in G$.

Output: $\alpha \in G$ such that $X^{\alpha}=Y$, or 'Fail' if $X$ and $Y$ are not conjugate.

(1) Using cyclings and decyclings, compute $X^{\prime} \in \operatorname{USS}(X)$ and $a \in G$ such that $X^{a}=X^{\prime}$.

(2) Using cyclings and decyclings, compute $Y^{\prime} \in \operatorname{USS}(Y)$ and $b \in G$ such that $Y^{b}=Y^{\prime}$.

(3) Using Algorithm 1, compute $\mathscr{B}_{X^{\prime}}$ and, for each vertex $V$ of $\mathcal{B}_{X^{\prime}}$, an element $c_{\left(X^{\prime}, V\right)}$ conjugating $X^{\prime}$ to $V$.

(4) Using Algorithm 2, compute $\mathscr{E}_{Y^{\prime}}$ and, for each vertex $V$ of $\mathscr{G}_{Y^{\prime}}$, an element $c_{\left(Y^{\prime}, V\right)}$ conjugating $Y^{\prime}$ to $V$.

(5) If $\mathcal{B}_{X^{\prime}} \cap \mathcal{E}_{Y^{\prime}}=\emptyset$ return 'Fail'.

(6) Choose $V \in \mathscr{B}_{X^{\prime}} \cap \mathscr{S}_{Y^{\prime}}$ and return $a c_{\left(X^{\prime}, V\right)} c_{\left(Y^{\prime}, V\right)}^{-1} b^{-1}$.

Therefore, in order to determine whether $X$ and $Y$ are conjugate, we just need to compute one black component and one grey component. The union of these two sets is in general smaller than the whole ultra summit set. Moreover, this procedure 
provides a conjugating element, since the algorithm computes graphs in such a way that we know how to join any two vertices in our graphs, and each path in the graph yields a conjugating element between the initial and the final vertex.

It should be mentioned that, in the worst case, this new algorithm is not better than the one in [18], since there are examples in which either $\mathcal{B}_{X^{\prime}}=\operatorname{USS}(X)$ or $\varepsilon_{Y^{\prime}}=\operatorname{USS}(Y)$. This is the case, for instance, for the periodic elements treated in Section 4.

5.3. Partial cycling subsumes decycling. In Corollary 2.10 we showed that we only need partial cyclings and partial twisted decyclings to connect a pair of elements in the same ultra summit set. We now prove that, given any $X \in G$, where in general $X \notin \operatorname{USS}(X)$, we can conjugate $X$ to an element in $\operatorname{USS}(X)$ by applying a finite number of partial cyclings.

We know by $[14, \S 4]$ and $[18, \S 1]$ (see also the review in [3]) that we can obtain an element in $\operatorname{USS}(X)$ from any $X \in G$ by iterated cyclings and decyclings. It is clear that a cycling is a particular case of a partial cycling, but we will now prove that a decycling can also be seen, in some sense, as a composition of several partial cyclings, provided that the element involved has maximal infimum in its conjugacy class.

Lemma 5.3. Let $X \in G$, and let $p=\inf (X)$ and $r=\ell(X)$. If the infimum of $X$ is maximal in its conjugacy class, then there exists a sequence $X=X_{1}, X_{2}, \ldots, X_{r}=$ $\mathbf{d}\left(\tau^{-p}(X)\right)$, such that $X_{i+1}$ is a partial cycling of $X_{i}$, for $i=1, \ldots, r-1$.

Proof. For simplicity, we will consider $Y=\tau^{-p}(X)$, and we will show that there is a sequence $\tau^{p}(Y)=Y_{1}, Y_{2}, \ldots, Y_{r}=\mathbf{d}(Y)$, consisting of partial cyclings. The claim is trivial for $r=0$, so assume $r>0$ and let $\Delta^{p} y_{1} \ldots y_{r}$ be the left normal form of $Y$. By definition, $\mathbf{d}(Y)=y_{r} \Delta^{p} y_{1} \ldots y_{r-1}=Y^{y_{r}^{-1}}$. Since $Y$ commutes with itself, one also has $\mathbf{d}(Y)=Y^{\left(Y y_{r}^{-1}\right)}=Y^{\left(\Delta^{p} y_{1} \ldots y_{r-1}\right)}=\left(\tau^{p}(Y)\right)^{\left(y_{1} \ldots y_{r-1}\right)}$.

Let $Y_{1}=\tau^{p}(Y)$ and $Y_{i+1}=Y_{i}^{y_{i}}$, for $i=1, \ldots, r-1$ (thus $\left.Y_{r}=\mathbf{d}(Y)\right)$. We will show that $Y_{i+1}$ is a partial cycling of $Y_{i}$ for $i=1, \ldots, r-1$.

Notice that for every element $Z$ such that $\inf (Z)=p$, the first factor in the left normal form of $Z \Delta^{-p}$ (whose infimum equals 0 ) is $\iota(Z)$. In other words, a simple element $s$ performs a partial cycling on $Z$ if and only if $s$ is a prefix of $Z \Delta^{-p}$.

Notice further that $Y_{1} \Delta^{-p}=\tau^{p}(Y) \Delta^{-p}=\Delta^{-p} Y=y_{1} \ldots y_{r}$ and that one hence has $Y_{i} \Delta^{-p}=Y_{1}^{\left(y_{1} \ldots y_{i-1}\right)} \Delta^{-p}=\left(y_{1} \ldots y_{i-1}\right)^{-1}\left(Y_{1} \Delta^{-p}\right) \tau^{-p}\left(y_{1} \ldots y_{i-1}\right)=$ $y_{i} \ldots y_{r} \tau^{-p}\left(y_{1} \ldots y_{i-1}\right)$ for every $i=2, \ldots, r-1$. Moreover, $\inf \left(Y_{i}\right)=p$, since it cannot be greater by hypothesis, and it cannot be smaller since $Y_{i} \Delta^{-p}$ is positive.

This implies that for $i=1, \ldots, r-1$ the simple factor $y_{i}$ is a prefix of $Y_{i} \Delta^{-p}$, where $\inf \left(Y_{i}\right)=p$. Therefore, $Y_{i+1}=Y_{i}^{y_{i}}$ is a partial cycling of $Y_{i}$ for $i=$ $1, \ldots, r-1$. 
Proposition 5.4. For $X \in G$, there exists a sequence $X=X_{1}, X_{2}, \ldots, X_{k}=Y$ in $G$, such that $Y \in \operatorname{USS}(X)$, and $X_{i+1}$ is a partial cycling of $X_{i}$ for $i=1, \ldots, k-1$.

Proof. It is well known [14, Lemma 4.3] that, by applying a finite sequence of cyclings to an element in $G$, one can achieve the maximal infimum in its conjugacy class. Since cyclings are a particular case of partial cyclings, we can assume that $X$ has maximal infimum in its conjugacy class.

By $[14, \S 4]$ and $[18, \S 1]$ we know that $\mathbf{c}^{s}\left(\mathbf{d}^{t}(X)\right) \in \operatorname{USS}(X)$, for some positive integers $s$ and $t$. On the other hand, we know that the ultra summit set is invariant under $\tau$. Hence, if we denote $p=\inf (X)$, we have $Y=\tau^{-p t}\left(\mathbf{c}^{s}\left(\mathbf{d}^{t}(X)\right)\right) \in$ $\operatorname{USS}(X)$.

By Lemma 2.4, $\tau$ commutes with cycling and decycling, hence we can write $Y=\mathbf{c}^{s}\left(\left(\mathbf{d} \circ \tau^{-p}\right)^{t}(X)\right) \in \operatorname{USS}(X)$. Finally, by Lemma 5.3, together with the fact that $X$ has maximal infimum in its conjugacy class, one can decompose every $\left(\mathbf{d} \circ \tau^{-p}\right)$ as a product of partial cyclings. Since the final application of cyclings also correspond to partial cyclings, the result follows.

5.4. An application to the theory of reductive groups. In $\S 4$ we showed that if $X$ is periodic and $\ell(X)>0$, then every arrow in $\Gamma_{X}$ is bi-colored. Hence $\Gamma_{X}=\mathcal{B}_{X}=\mathscr{E}_{X}$. In particular, every two elements in $\operatorname{USS}(X)$ can be connected by a sequence of partial cyclings.

It was communicated to us by Jean Michel that this has important consequences in the theory of reductive groups. Namely, in a reductive group, the Deligne-Lusztig varieties are related to the elements of the braid monoid of the Weil group $W$. In particular, the Deligne-Lusztig varieties which appear in Broué's conjecture correspond to periodic elements in an Artin-Tits group. There is a conjecture which states that there is essentially a unique Deligne-Lusztig variety for each period. François Digne and Jean Michel have shown that, if two periodic elements are conjugate by a partial cycling, then their corresponding Deligne-Lusztig varieties are essentially the same. In the case of Artin braid groups (which corresponds to the linear algebraic groups), it is known that two periodic elements of the same period are conjugate [19, Theorem 1.1]. Hence, the results of Section 4 show that the above conjecture is true, at least for Artin braid groups (Artin-Tits groups of type A). In order to show the conjecture in general, it remains to be shown that every two periodic elements of the same period, in any spherical type Artin-Tits group, are conjugate. After this paper was submitted, David Bessis solved this problem in [1], Theorem 2.15. Hence the results in Section 4, together with Theorem 2.15 of [1], show the above conjecture in its general setting. We refer to [12] for details on this problem. 


\section{References}

[1] D. Bessis, Finite complex reflection arrangements are $K(\pi, 1)$. Preprint, 2007. arXiv:math/0610777v3.

[2] M. Bestvina, Non-positively curved aspects of Artin groups of finite type. Geom. Topol. 3 (1999), 269-302. Zbl 0998.20034 MR 1714913

[3] J. S. Birman, V. Gebhardt and J. González-Meneses, Conjugacy in Garside groups I: cycling, powers and rigidity. Groups Geom. Dyn. 1 (2007), 221-279. MR 2314045

[4] J. S. Birman, V. Gebhardt and J. González-Meneses, Conjugacy in Garside groups III: Periodic braids. J. Algebra 316 (2007), 746-776.

[5] J. S. Birman, K. H. Ko, and S. J. Lee, A new approach to the word and conjugacy problems in the braid groups. Adv. Math. 139 (1998), 322-353. Zbl 0937.20016 MR 1654165

[6] J. S. Birman, K. H. Ko, and S. J. Lee, The infimum, supremum, and geodesic length of a braid conjugacy class. Adv. Math. 164 (2001), 41-56. Zbl 1063.20039 MR 1870512

[7] E. Brieskorn and K. Saito, Artin-Gruppen und Coxeter-Gruppen. Invent. Math. 17 (1972), 245-271. Zbl 0243.20037 MR 0323910

[8] R. Charney, Artin groups of finite type are biautomatic. Math. Ann. 292 (1992), 671-683. Zbl 0736.57001 MR 1157320

[9] R. Charney, J. Meier and K. Whittlesey, Bestvina's normal form complex and the homology of Garside groups. Geom. Dedicata 105 (2004), 171-188. Zbl 1064.20044 MR 2057250

[10] A. Constantin and B. Kolev, The theorem of Kerékjártó on periodic homeomorphisms of the disc and the sphere. Enseign. Math. 40 (1994), 193-204. Zbl 0852.57012 MR 1309126

[11] P. Dehornoy and L. Paris, Gaussian groups and Garside groups, two generalisations of Artin groups. Proc. London Math. Soc. (3) 79 (1999), 569-604. Zbl 1030.20021 MR 1710165

[12] F. Digne and J. Michel, Endomorphisms of Deligne-Lusztig varieties. Nagoya Math. J. 183 (2006), 35-103. Zbl 1119.20008 MR 2253886

[13] S. Eilenberg, Sur les transformations périodiques de la surface de la sphère. Fund. Math. 22 (1934), 28-41. Zbl 0008.37109

[14] E. Elrifai and H. Morton, Algorithms for positive braids. Quart. J. Math. Oxford Ser. (2) 45 (1994), 479-497. Zbl 0839.20051 MR 1315459

[15] D. B. A. Epstein, J. W. Cannon, D. F. Holt, S. V. F. Levy, M. S. Paterson, and W. P. Thurston, Word processing in groups. Jones and Bartlett Publishers, Boston 1992. Zbl 0764.20017 MR 1161694

[16] N. Franco and J. González-Meneses, Conjugacy problem for braid groups and Garside groups. J. Algebra 266 (2003), 112-132. Zbl 1043.20019 MR 1994532

[17] F. Garside, The braid group and other groups. Quart. J. Math. Oxford Ser. (2) 20 (1969), 235-254. Zbl 0194.03303 MR 0248801

[18] V. Gebhardt, A new approach to the conjugacy problem in Garside groups. J. Algebra 292 (2005), 282-302. Zbl 1105.20032 MR 2166805 
[19] J. González-Meneses, The $n$th root of a braid is unique up to conjugacy. Algebr. Geom. Topol. 3 (2003), 1103-1118. Zbl 1063.20041 MR 2012967

[20] B. von Kerékjártó, Über die periodischen Transformationen der Kreisscheibe und der Kugelfläche. Math. Ann. 80 (1919), 36-38. JFM 47.0526.05

[21] J. Michel, A note on words in braid monoids. J. Algebra 215 (1999), 366-377. Zbl 0937.20017 MR 1684142

[22] W. Thurston, On the geometry and dynamics of diffeomorphisms of surfaces. Bull. Amer. Math. Soc. (N.S.) 19 (1988), 417-431. Zbl 0674.57008 MR 956596

Received October 12, 2006; revised January 27, 2007

J. S. Birman, Department of Mathematics, Barnard College and Columbia University, 2990 Broadway, New York, New York 10027, U.S.A.

E-mail: jb@math.columbia.edu

V. Gebhardt, School of Computing and Mathematics, University of Western Sydney, Locked Bag 1797, Penrith South DC NSW 1797, Australia

E-mail: v.gebhardt@uws.edu.au

J. González-Meneses, Departamento de Álgebra, Universidad de Sevilla, Apdo. 1160, 41080 Sevilla, Spain

E-mail: meneses@us.es 